\title{
Symmetric Simplicial Pseudoline Arrangements
}

\author{
Leah Wrenn Berman \\ Ursinus College \\ Department of Mathematics and Computer Science \\ P.O. Box 1000 \\ Collegeville, PA 19426 \\ USA \\ lberman@ursinus.edu
}

Submitted: May 11, 2007; Accepted: Oct 21, 2007; Published: Jan 14, 2008

Mathematics Subject Classification: 52C30

\begin{abstract}
A simplicial arrangement of pseudolines is a collection of topological lines in the projective plane where each region that is formed is triangular. This paper refines and develops David Eppstein's notion of a kaleidoscope construction for symmetric pseudoline arrangements to construct and analyze several infinite families of simplicial pseudoline arrangements with high degrees of geometric symmetry. In particular, all simplicial pseudoline arrangements with the symmetries of a regular $k$-gon and three symmetry classes of pseudolines, consisting of the mirrors of the $k$-gon and two other symmetry classes, plus sometimes the line at infinity, are classified, and other interesting families (with more symmetry classes of pseudolines) are discussed.
\end{abstract}

\section{Introduction and Definitions}

An arrangement of lines is any finite family of lines in the projective plane [9, p. 4]. Such a family of lines partitions the plane into regions. If all the regions are triangular, the arrangement is said to be simplicial. The current state of knowledge about linear simplicial arrangements has been summarized in Grünbaum's survey [7], which is an update of results from [10].

Arrangements may be generalized by replacing the straight lines with pseudolines [9, sections 3.1-3.2]. In the projective plane, a pseudoline is a simple closed curve that is topologically equivalent to a line [9, p. 40]. In the Euclidean plane, every pseudoline may be represented by a straight line that has been modified in a piecewise-linear fashion in a finite part so as to remain simple. A family of pseudolines has the additional restriction imposed that given any two pseudolines, either the infinite parts are parallel and the two 
pseudolines are disjoint, or the two pseudolines cross each other at a single point; that is, even though they may wiggle around somewhat, the pseudolines should 'behave like lines'.

In addition to simplicial arrangements of lines or pseudolines being of inherent geometric interest, there are also some interesting connections between simplicial arrangements and other mathematical objects, and they often provide interesting examples or counterexamples to questions in the study of arrangements. Pseudolines serve as geometric representations of rank 3 oriented matroids and have been studied extensively [6]. One recent publication [5] discusses a method for constructing cubic partial cubes, useful in some areas of computer science, using simplicial arrangements of lines or pseudolines. Another application is to invariant lines in certain differential systems [1].

By a symmetric pseudoline arrangement we mean an arrangement of pseudolines with non-trivial geometric (that is, Euclidean) symmetry. For this notion to make sense, we must explicitly be working in the 'extended Euclidean plane' model of the projective plane; that is, we are in the Euclidean plane, but there is an extra line at infinity, and pencils of parallel lines meet at a point on the line at infinity corresponding to the angle the parallel lines make with horizontal. We say two pseudolines are parallel if they are disjoint in their finite parts and have parallel infinite parts. If the line at infinity is viewed as a circle, then antipodal points on the circle are identified. Figure 1 shows several examples of linear arrangements in the extended Euclidean plane. Figure 1(a) shows a simplicial arrangement; the shaded (lavender) region is triangular, and the line at infinity, which is indicated by a dashed circle because it is not part of the arrangement, passes through it. Figure 1(b) shows the same simplicial arrangement with the line at infinity included, in this case indicated by a thick circle. In this diagram, both shaded regions (colored lavender and yellow) are triangular and have part of the line at infinity as an edge. Note that arrangements which are simplicial in the finite part of the plane that have multiple pencils of parallel (pseudo)lines not separated by single (pseudo)lines require the inclusion of the line at infinity in order to be simplicial. Figure 1(c) shows an arrangement of lines, simplicial in its finite part, which is not simplicial, because the shaded region is a quadrilateral (it has four vertices colored red, green, cyan and yellow). Adding in the line at infinity as a line in the arrangement converts it to the simplicial arrangement $\mathcal{A}(10,3)$ in [7. In subsequent examples, if the line at infinity must be included in the arrangement, it will be indicated by $\infty$ next to the arrangement, which will be drawn in the ordinary Euclidean plane, rather than drawing the arrangement schematically, with the line at infinity indicated by an outer circle. Many of the arrangements which are not required to include the line at infinity may include it; optional inclusion will be indicated by $(\infty)$. If neither notation appears in a diagram, then the line at infinity may not be included as a line of the arrangement.

There has been no systematic study of symmetric simplicial pseudoline arrangements, although isolated examples of symmetric pseudoline arrangements have appeared here and there. Grünbaum provided several examples in his monograph Arrangements and Spreads [9, Figures 3.15 - 3.18], there are a few more given by Eppstein in [3, 4, 5], and two examples are given in [6. Figures 5.4.1, 5.4.2]. In addition, there are a few examples 


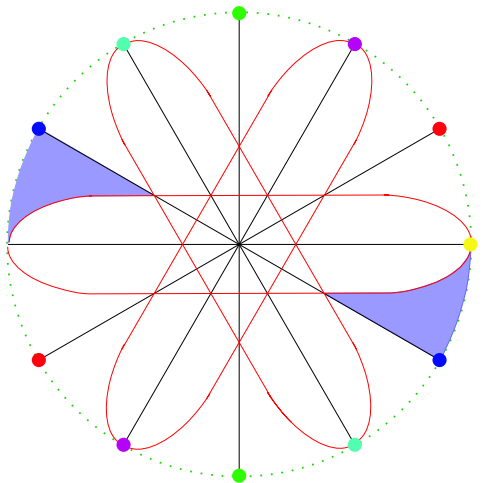

(a)

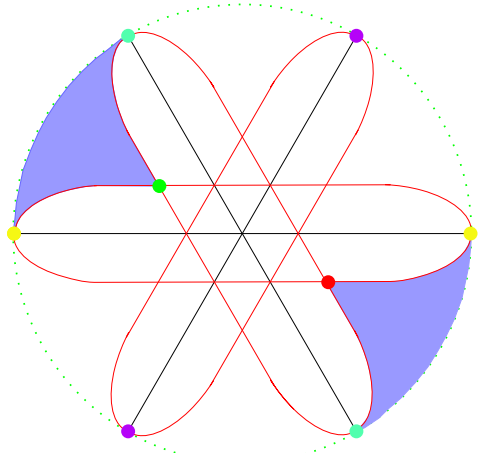

(c)

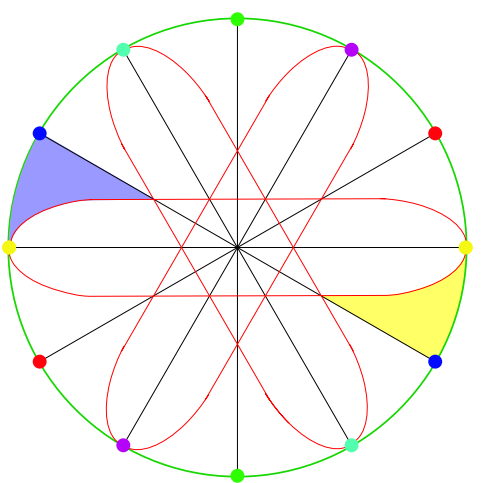

(b)

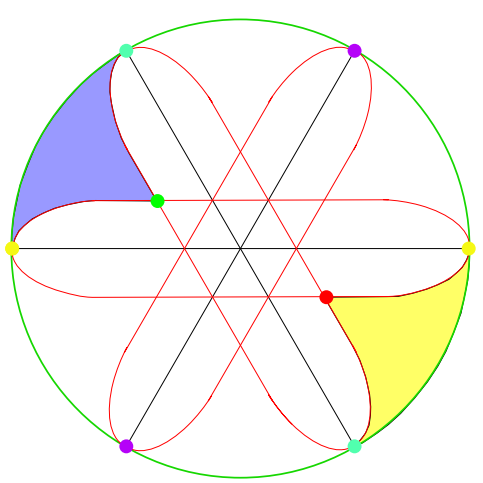

(d)

Figure 1: Schematic drawings of linear arrangements in the extended Euclidean plane; the outer circle represents the line at infinity. Points of the same color are identified, and arrangement labels are taken from [7]. (a) The linear simplicial arrangement $\mathcal{A}(12,1)$, excluding the line at infinity (dashed); (b) The linear simplicial arrangement $\mathcal{A}(13,1)$, including the line at infinity (thick green circle); (c) A non-simplicial arrangement of lines that does not include the line at infinity (the shaded region is a quadrilateral); (d) including the line at infinity forms the simplicial arrangement $\mathcal{A}(10,3)$. 
of symmetric pseudoline configurations, such as those listed in [2] and [8, which may be viewed as simplicial pseudoline arrangements when the mirrors of the configuration are included. In Arrangements and Spreads [9, p. 51], Grünbaum mentioned the existence of seven infinite families of simplicial pseudoline arrangements (also alluded to in [7]), but details of these families have not been published.

This paper will produce several infinite families of symmetric simplicial pseudoline arrangements with $k$-gonal symmetry. (In a private conversation with Grünbaum, the author has verified that all the infinite families of simplicial arrangements known to Grünbaum are accounted for in the infinite families produced in this paper, although the families are divided differently.) In Section 2, we introduce and analyze a method of constructing symmetric pseudoline arrangements, by viewing the mirrors of a regular $k$-gon as actual mirrors and reflecting a beam kaleidoscopically to create pseudolines. Sections 3 and 4 use this method to classify all simplicial pseudoline arrangements constructed from the mirrors of a regular $k$-gon and one or two additional symmetry classes of pseudolines (in sections 3 and 4 respectively). Section 5 develops two infinite classes of symmetric simplicial pseudoline arrangements constructed from the mirrors of a regular $k$-gon and three additional symmetry classes of pseudolines. Finally, Section [6 discusses symmetric simplicial pseudoline arrangements with many symmetry classes of pseudolines and also interprets known linear simplicial arrangements as kaleidoscope arrangements.

\section{Kaleidoscope analysis}

On his blog [3], David Eppstein briefly sketched a method of looking at symmetric pseudoline arrangements that is very useful, both in analyzing a given arrangement and in constructing new arrangements; I have refined and further developed his short description in what follows.

We are interested in the construction of symmetric simplicial pseudoline arrangements with the symmetries of a regular $k$-gon. To construct such an arrangement, do the following.

Take $2 k$ rays with a common endpoint at the origin $\mathcal{O}$ arranged so that the angle between them is $\frac{\pi}{k}$; think of these as forming the mirrors of a kaleidoscope with $k$-gonal symmetry. Bounce between two consecutive rays, the bounding mirrors, a sequence of line "segments" (the first segment is actually a particularly chosen ray), where consecutive segments share the same endpoint; we will call this sequence a beam (Figure 2(a)). We will reflect the beam kaleidosopically by reflecting it (geometrically) consecutively over all $2 k$ mirrors (see Figure 2(b)). Note that the result is the same as what you would see if the bounding mirrors were actual mirrors and you looked into them, as in a kaleidoscope. By convention, we will assume the bounding mirrors are the horizonal ray $r_{0}$, beginning at $\mathcal{O}$ and passing through $(1,0)$, and the ray $r_{1}$ which is rotation of $r_{0}$ about $\mathcal{O}$ by $\frac{\pi}{m}$; the remaining $2 k$ rays will be labelled $r_{2}, \ldots, r_{2 k-1}$, where ray $r_{i}$ is the rotation of $r_{0}$ through $\frac{i \pi}{k}$ about the origin.

Note that in fact, these line "segments" may in fact themselves be pseudosegments and pseudorays (that is, parts of a pseudoline, not necessarily straight), necessary for 
construction of the three-beam families of arrangements discussed in Section [5, but the only places they are allowed to intersect the bounding mirrors are at the endpoints of the pseudosegments or pseudorays. In this case, the reflections through the bounding mirrors are taken to be geometric reflections through lines or rays and may not obey rules for optical reflection.

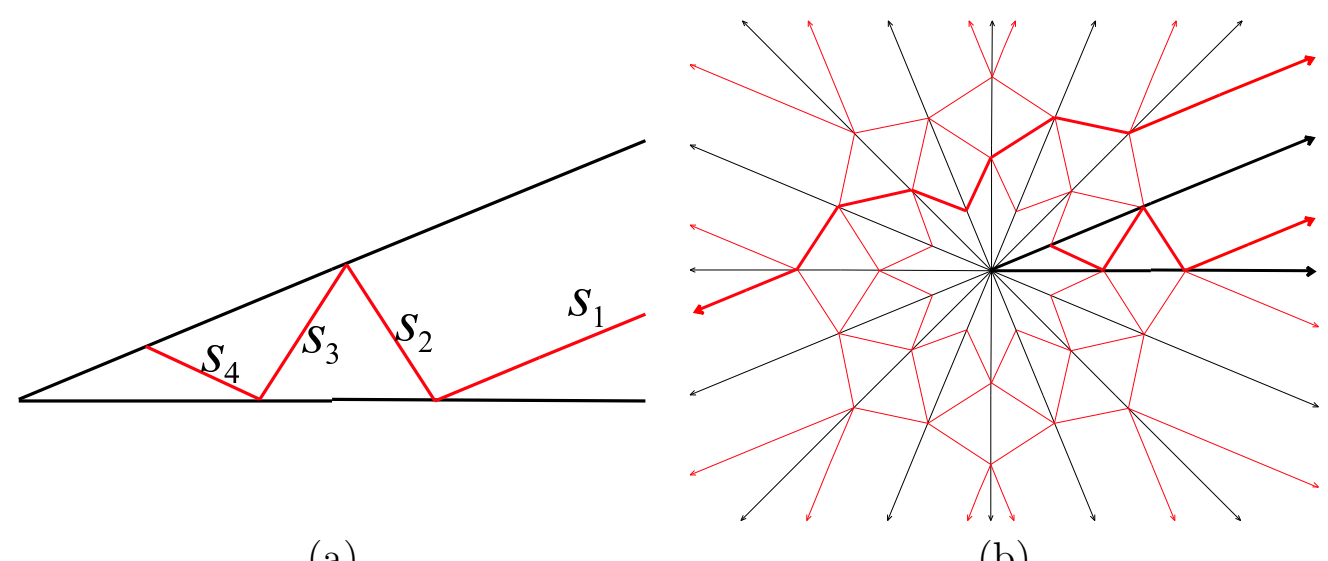

(a)

(b)

Figure 2: Here, $k=8$. (a) An example of a beam; (b) The beam reflected kaleidosopically. Here, both the beam and a resulting pseudoline are indicated with thick lines.

If the beam obeys certain constraints, then after it is reflected kaleidoscopically, the result will be a collection of $k$ pseudolines, which by construction are in the same symmetry class; we will say these pseudolines are generated by the beam. Since we may assume that every pseudoline is piecewise linear and has its non-linear part restricted to a finite region (in our case bounded by a circle centered at $\mathcal{O}$ ), we need the initial ray of the beam to be coincident with the line formed by an extended side of a regular $k$-gon.

\subsection{Pseudoline generation from a beam}

Let the beam segments have labels $s_{1}, s_{2}, \ldots, s_{b}$, with $s_{1}$ the initial ray of the beam, and label the rays (mirrors) as $r_{0}, \ldots, r_{2 k-1}$ as above, where $r_{0}$ and $r_{1}$ are the bounding mirrors. Informally, to construct an individual pseudoline, the idea is to march down the beam and then back up, reflecting each beam segment over a ray or reflecting it over two rays (that is, rotating it), while consecutively shifting the rays participating in the reflection or rotation. For example, in the case shown in Figure 3 where $s_{1}$ intersects $r_{0}$, marching down the beam, first $s_{1}$ is reflected over $r_{1}$, then $s_{2}$ is reflected over $r_{1}$ followed by $r_{2}$, then $s_{3}$ is reflected over $r_{2}$, and $s_{4}$ is reflected over $r_{1}$ and then $r_{3}$. Marching back up the beam, $s_{4}$ is reflected over $r_{3}$, then $s_{3}$ is reflected over $r_{1}$ and then $r_{4}, s_{2}$ is reflected over $r_{4}$, and finally, $s_{1}$ is reflected over $r_{1}$ and then $r_{5}$. Developing this process in general takes a fair amount of careful accounting.

Suppose that $s_{1} \cap r_{w}$, where $w \in\{0,1\}$ (that is, we define $w=0$ if the head of the initial ray $s_{1}$ lies on ray $r_{0}$, and likewise $w=1$ if the head of $s_{1}$ lies on $r_{1}$ ). Define $s_{i}^{j}$ to 
be the image of $s_{i}$ reflected over mirror $r_{j}$ and $s_{i}^{j, q}$ to be the image of $s_{i}$ reflected first over mirror $r_{j}$ and then over $r_{q}$. Finally, define

$$
f_{i j}=\left\lfloor\frac{i+2 j+1+\bmod (i+w+1,2)}{2}\right\rfloor .
$$

We define a pseudoline $P^{j}$ to be the collection of segments

$$
p_{i}^{j}= \begin{cases}s_{i}^{1, f_{i j}} & \text { if } 1 \leq i \leq b \text { and } i+w \text { is even } \\ s_{i}^{f_{i j}} & \text { if } 1 \leq i \leq b \text { and } i+w \text { is odd } \\ s_{2, f_{i j}} & \text { if } b<i \leq 2 b \text { and } i+w \text { is even } \\ s_{2 b-i+1} & \text { if } b<i \leq 2 b \text { and } i+w \text { is odd }\end{cases}
$$

Since the composition of two reflections is a rotation, segments of the form $s_{i}^{1, f_{i j}}$ are rotations of segment $s_{i}$, while segments of the form $s_{i}^{f_{i j}}$ are reflections. Thus, equation (11) says that the beam segments are alternately reflected and rotated as you march down the beam along $s_{1}, s_{2}, \ldots, s_{b}$ and then back up along $s_{b}, s_{b-1} \ldots, s_{1}$.

The potential pseudoline $P^{j}$ is formed by the collection of segments $p_{1}^{j}, p_{2}^{j} \ldots, p_{2 b}^{j}$ (see Figure 3).

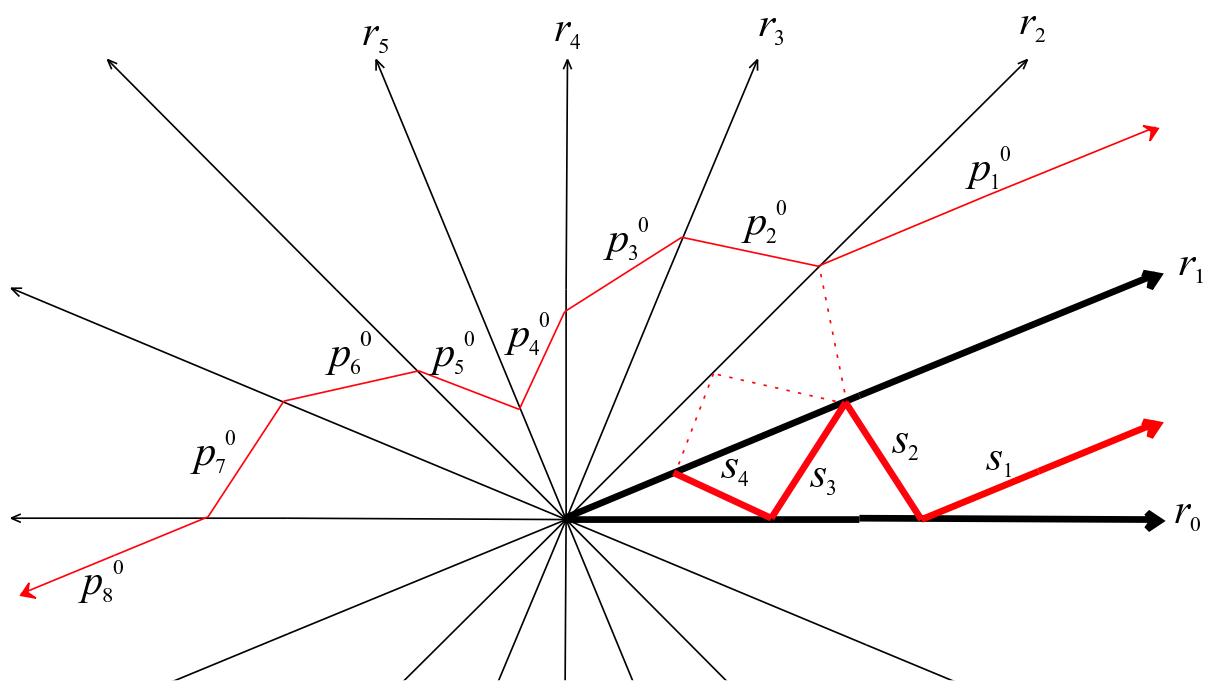

Figure 3: A labelled pseudoline $P^{0}$ formed by reflecting a beam according to equation (11); $k=8, b=4, w=0$.

Theorem 1. Suppose $\mathcal{B}=\left\{s_{1}, s_{2}, \ldots, s_{b}\right\}$ is a beam that generates pseudolines $P^{0}, \ldots$, $P^{k-1}$. If $k$ is even, the initial ray $s_{1}$ must be parallel to one of the bounding mirrors, and if $k$ is odd, $s_{1}$ must be parallel to the angle bisector of the two bounding mirrors. Moreover, $b=\left\lfloor\frac{k+1}{2}\right\rfloor$, to ensure that after the beam is reflected kaleidosopically, the resulting pseudolines differ from a straight line only in a finite part. 
Proof. By convention, the lower bounding mirror $r_{0}$ is the horizontal ray passing through $\mathcal{O}$ and $(1,0)$ and the other mirrors $r_{i}$, for $i=1, \ldots, 2 k-1$ are the image of $r_{0}$ through rotation by $\frac{\pi i}{k}$ about the origin, with $r_{1}$ as the upper bounding mirror.

To verify that the initial ray must be parallel as stated, notice that symmetry constraints force the infinite parts of the collection of (potential) pseudolines to coincide with the extended sides of the regular $k$-gon whose vertices are formed by the $k$-fold rotation of the head of $s_{1}$, since otherwise, each potential pseudoline would not coincide with some line outside of a finite area. If $k$ is even, regular $k$-gons with vertices generated by a point on some $r_{i}$ have extended sides parallel to the mirrors through the origin with angle $\frac{j \pi}{k}$ for odd $j$, while if $k$ is odd, such regular $k$-gons have extended sides parallel to the mirrors through the origin with angle $\frac{j \pi}{2 k}$ for odd $j$. Since the angle between the bounding mirrors for the beam is $\frac{\pi}{k}$, if $k$ is odd, the ray with angle $\frac{\pi}{2 k}$ is the angle bisector of the two bounding mirrors.

Suppose that the beam $\mathcal{B}=\left\{s_{1}, \ldots, s_{b}\right\}$, where $s_{1}$ is the initial ray, and suppose that the originating vertex of $s_{1}$ lies on the ray $r_{w}$, where $w$ is either 0 or 1 . To see that the beam must have precisely $\left\lfloor\frac{k+1}{2}\right\rfloor$ segments, by symmetry, it suffices to consider the purported pseudoline $P^{0}=\left\{p_{i}^{0}\right\}_{i=1}^{2 b}$.

Because far away from the origin the pseudoline arrangement must behave like a line arrangement, $P^{0}$ must be mirror-symmetric and $p_{2 b}^{0}$ must lie on the line generated by the ray $p_{1}^{0}$.

There are two cases to consider, where $w=0$ and $w=1$; in each case, we will consider separately the situation when $k$ is even and when $k$ is odd (since the rays $s_{1}$ in the even and odd case are different).

First, suppose $w=1$. By applying equation (1), $p_{1}^{0}=s_{1}^{1,1}=s_{1}$ and $p_{2 b}^{0}=s_{1}^{b}$. If $k$ is even, the line containing $p_{1}^{0}$ is parallel to $r_{0}$, so the perpendicular line to $p_{1}^{0}$ makes an angle with horizontal of $\frac{\pi}{2}=\frac{k}{2}\left(\frac{\pi}{k}\right)$; that is, $r_{k / 2}$ is perpendicular to $p_{1}^{0}$. In order for the pseudoline $P^{0}$ to differ from the line containing $p_{1}^{0}$ only in a finite part, $p_{2 b}^{0}$ must also lie on this line; that is, $p_{2 b}^{0}$ should be the reflection of $p_{1}^{0}$ through the ray $r_{k / 2}$. Since $p_{2 b}^{0}$ is the reflection of $s_{1}=p_{1}^{0}$ through the ray $r_{b}$ (by the definition of the notation $s_{1}^{b}$ ), it follows that $b=\frac{k}{2}=\left\lfloor\frac{k+1}{2}\right\rfloor$.

If $k$ is odd and $w=1$, then the line containing $p_{1}^{0}$ is parallel to the angle bisector of $r_{0}$ and $r_{1}$, which makes an angle of $\frac{\pi}{2 k}$ with horizontal. Therefore, the perpendicular line to $p_{1}^{0}$ makes an angle with horizontal of $\frac{\pi}{2}+\frac{\pi}{2 k}=\frac{k+1}{2}\left(\frac{\pi}{k}\right)$; that is $r_{(k+1) / 2}$ is perpendicular to $p_{1}^{0}$. Hence $b=\frac{k+1}{2}=\left\lfloor\frac{k+1}{2}\right\rfloor$.

The analysis is similar in the case where $w=0$. In this case, $p_{1}^{0}=s_{1}^{1}$ and $p_{2 b}^{0}=s_{1}^{1, b+1}$; note that $s_{1}^{1, b+1}$ is the reflection of $s_{1}^{1}$ through the ray $r_{b+1}$. That is, $p_{2 b}^{0}$ is the reflection of $p_{1}^{0}$ through ray $r_{b+1}$. If $k$ is even, the perpendicular to $p_{1}^{0}=s_{1}^{1}$ makes an angle with horizontal of $\frac{\pi}{2}+\frac{\pi}{k}=\frac{k+2}{2}\left(\frac{\pi}{k}\right)$, so $b+1=\frac{k+2}{2}$ and hence $b=\frac{k}{2}=\left\lfloor\frac{k+1}{2}\right\rfloor$. On the other hand, if $k$ is odd, then the perpendicular to $p_{1}^{0}=s_{1}^{1}$ makes an angle with horizontal of $\frac{\pi}{2}+\frac{\pi}{k}+\frac{\pi}{2 k}=\frac{k+3}{2}\left(\frac{\pi}{k}\right)$, so $b+1=\frac{k+3}{2}$ and therefore $b=\frac{k+1}{2}=\left\lfloor\frac{k+1}{2}\right\rfloor$.

To construct a linear arrangement, the beam has to behave as though it came from an 
actual laser (see Figure 4), in that the sequence of line segments needs to begin perpendicularly from one of the mirrors and as it bounces back and forth, the angle of incidence must equal the angle of reflection for each pair of consecutive segments.
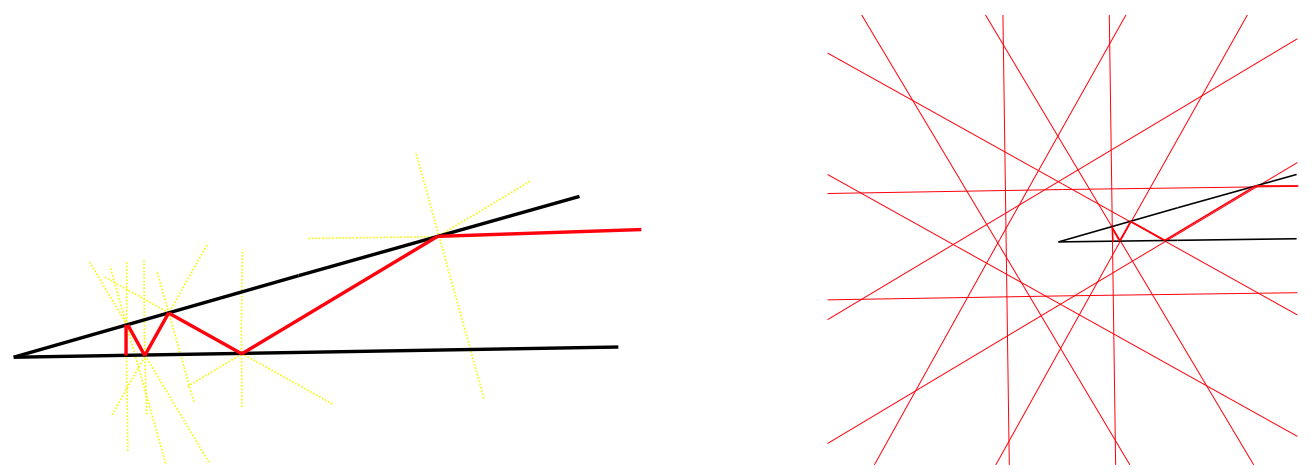

Figure 4: A beam that behaves like a laser, corresponding to an arrangement of lines.

\section{Symmetric arrangements using a single beam}

Consider the pseudoline arrangement formed by the mirrors (considered as lines of the arrangement) of a regular $k$-gon along with the pseudolines generated by a single beam of length $\left\lfloor\frac{k+1}{2}\right\rfloor$. Such an arrangement is well-known; it is isomorphic to a regular $k$ gon plus its mirrors of symmetry. As mentioned by Grünbaum in [9, p. 9], a regular $k$-gon plus its axes of symmetry forms a (linear) simplicial arrangement, denoted there as $\mathcal{R}(2 k)$; in [7] such arrangements are listed as $\mathcal{A}(2 k, 1)$. Figure 1(a) shows the arrangement $\mathcal{R}(12)=\mathcal{A}(12,1)$. If the line at infinity is included, as in Figure 1(b), which is possible when $k$ is even, the arrangements in general are denoted as $\mathcal{R}(4 m+1)=\mathcal{A}(4 m+1,1)$; Figure 1(b) shows $\mathcal{R}(13)=\mathcal{A}(13,1)$.

\section{Two beams}

Here we will classify all simplicial arrangements of pseudolines formed by the pseudolines generated by two beams, plus the mirrors. Note that this completely classifies all simplicial pseudoline arrangements with $k$-gonal symmetry and three symmetry classes of pseudolines, where one of the classes consists of the mirrors of the arrangement.

From Theorem 1, we know that each beam must consist of $b=\left\lfloor\frac{k+1}{2}\right\rfloor$ line segments, including an initial ray which is parallel to the opposite bounding mirror or the angle bisector of the bounding mirrors, depending on if $k$ is even or odd. We are interested in producing simplicial arrangements, so there are two questions to ask: (i) how can the beams interact so that the generated pseduolines do not intersect each other multiple times (that is, so the pseudolines generated $d o$ form an arrangement of pseudolines), and (ii) how can the beams intersect so that the resulting arrangement is simplicial. 


\subsection{Two beams crossing}

Recall that a beam $\mathcal{B}=\left\{s_{1}, \ldots, s_{b}\right\}$ generates a pseudoline

$$
P^{j}=\left\{p_{1}^{j}, \ldots, p_{b}^{j}, p_{b+1}^{j}, \ldots, p_{2 b}^{j}\right\}
$$

where $p_{i}^{j}$ is defined according to equation (11). Define $\mathcal{S}^{i}$ to be the sector of the plane enclosed by rays $r_{i}$ and $r_{i+1}$; then

$$
p_{i}^{j} \in \mathcal{S}^{i+2 j-w},
$$

where $s_{1} \cap r_{w}$.

We will call segments $p_{1}^{j}, \ldots, p_{b}^{j}$ the front of the pseudoline $P^{j}$ and segments $p_{b+1}^{j}, \ldots$, $p_{2 b}^{j}$ the back of the pseudoline. A single beam segment $s_{i}$ generates two segments in each pseudoline, $p_{i}^{j}$ in the front and $p_{2 b-i+1}^{j}$ in the back. We will use this front/back notion to help classify the kinds of intersections between pseudolines generated by two beams, one green and one red.

Suppose we have two beams: a red beam $\mathcal{B}_{r}=\left\{\left(s_{r}\right)_{1}, \ldots,\left(s_{r}\right)_{b}\right\}$ and a green beam $\mathcal{B}_{g}=\left\{\left(s_{g}\right)_{1}, \ldots,\left(s_{g}\right)_{b}\right\}$. Suppose that $\left(s_{r}\right)_{1} \cap r_{w_{r}}$ and $\left(s_{g}\right)_{1} \cap r_{w_{g}}$, where $w_{r}$ and $w_{g}$ are either 0 or 1 . These beams will intersect each other $b$ times.

If $\left(s_{r}\right)_{i}$ intersects $\left(s_{g}\right)_{q}$, then we will denote this as $\left(s_{r}\right)_{i} \times\left(s_{g}\right)_{q}$. To determine whether the potential pseudolines intersect appropriately, by symmetry, it suffices to consider the intersections formed as a result of this intersection between the 0 -th red pseudoline $\left(P_{r}\right)^{0}$ and some green pseudoline $\left(P_{g}\right)^{j}$.

Case 1: front/front intersection. In this case, we suppose that the front segment generated by $\left(s_{r}\right)_{i}$ in the red pseudoline $\left(P_{r}\right)^{0}$ is intersected by the front segment generated by $\left(s_{g}\right)_{q}$ in the green pseudoline $\left(P_{g}\right)^{j}$. In order for the two pseudolines to intersect, the green pseudoline segment $\left(p_{g}\right)_{q}^{j}$ must lie in the same sector as the red pseudoline segment $\left(p_{r}\right)_{i}^{0}$.

Since

$$
\left(p_{r}\right)_{i}^{0} \in \mathcal{S}^{i-w_{r}} \quad \text { and } \quad\left(p_{g}\right)_{q}^{j} \in \mathcal{S}^{q+2 j-w_{g}},
$$

it must be the case that

$$
\begin{aligned}
i-w_{r} & =q+2 j-w_{g}, \text { so } \\
2 j & =(i-q)+\left(w_{g}-w_{r}\right) .
\end{aligned}
$$

Since $j$ must be an integer, in order to have front/front intersection, it follows that

$$
i-q \equiv w_{g}-w_{r} \bmod 2
$$

and therefore

$$
j=\frac{(i-q)+\left(w_{g}-w_{r}\right)}{2} .
$$


Case 2: back/back intersection. In this case, we suppose that the back segment generated by $\left(s_{r}\right)_{i}$ in the red pseudoline $\left(P_{r}\right)^{0}$ is intersected by the back segment generated by $\left(s_{g}\right)_{q}$ in the green pseudoline $\left(P_{g}\right)^{j}$. In order for the two pseudolines to intersect, the green pseudoline segment $\left(p_{g}\right)_{2 b-q+1}^{j}$ must lie in the same sector as the red pseudoline segment $\left(p_{r}\right)_{2 b-i+1}^{0}$.

In this case, matching sectors as above, we see that

$$
\begin{aligned}
2 b-q+1+2 j-w_{g} & =2 b-i+1-w_{r}, \text { so } \\
2 j & =(q-i)+\left(w_{g}-w_{r}\right) .
\end{aligned}
$$

Since $j$ must be an integer, again

$$
i-q \equiv w_{g}-w_{r} \bmod 2,
$$

but here,

$$
j=\frac{-(i-q)+\left(w_{g}-w_{r}\right)}{2} .
$$

(Note that values for $j$ are taken modulo $k$.)

Case 3: front/back intersection. In this case, we suppose that the front segment generated by $\left(s_{r}\right)_{i}$ in the red pseudoline $\left(P_{r}\right)^{0}$ is intersected by the back segment generated by $\left(s_{g}\right)_{q}$ in the green pseudoline $\left(P_{g}\right)^{j}$, so the green pseudoline segment $\left(p_{g}\right)_{2 b-q+1}^{j}$ must lie in the same sector as the red pseudoline segment $\left(p_{r}\right)_{i}^{0}$.

Therefore,

$$
\begin{aligned}
2 b-q+1+2 j-w_{g} & =i-w_{r}, \text { so } \\
2 j & =(i+q)+\left(w_{g}-w_{r}\right)-2 b-1 .
\end{aligned}
$$

Since $j$ must be an integer, it must be that

$$
i-q \not \equiv w_{g}-w_{r} \bmod 2 .
$$

and

$$
j=\frac{(i+q)+\left(w_{g}-w_{r}\right)-1}{2}-b .
$$

Case 4: back/front intersection. In this case, we suppose that the back segment generated by $\left(s_{r}\right)_{i}$ in the red pseudoline $\left(P_{r}\right)^{0}$ is intersected by the front segment generated by $\left(s_{g}\right)_{q}$ in the green pseudoline $\left(P_{g}\right)^{j}$, so the green pseudoline segment $\left(p_{g}\right)_{q}^{j}$ must lie in the same sector as the red pseudoline segment $\left(p_{r}\right)_{2 b-i+1}^{0}$.

Therefore,

$$
\begin{aligned}
q+2 j-w_{g} & =2 b-i+1-w_{r}, \text { so } \\
2 j & =-(i+q)+\left(w_{g}-w_{r}\right)+2 b+1 .
\end{aligned}
$$


Since $j$ must be an integer,

$$
i-q \not \equiv w_{g}-w_{r} \bmod 2
$$

and

$$
j=-\left(\frac{(q-i)-\left(w_{g}-w_{r}\right)-1}{2}-b\right) .
$$

In order to determine what kinds of crossings are inadmissible between the beams, we can suppose that we have two crossings $\left(s_{r}\right)_{i} \times\left(s_{g}\right)_{q}$ and $\left(s_{r}\right)_{i^{\prime}} \times\left(s_{g}\right)_{q^{\prime}}$. Moreover, these crossings must generate two pseudoline crossings between $\left(P_{r}\right)^{0}$ and $\left(P_{g}\right)^{j}$ (since this would invalidate the corresponding pseudoline arrangement). Each crossing must be one of the four cases, above. Straightforward combinatorics would suggest that there are 16 possible combinations of beams with two crossings to consider. However, we can limit our cases somewhat by observing that the order of the pairings does not matter (it corresponds to switching the role of $i$ and $i^{\prime}$ ) and interchanging "front" crossings with "back" crossings corresponds to switching the red and green beams. Thus, we have six cases to consider. Five of them turn out to be possible and lead to badly intersecting pseudolines.

Case I: front/front and front/front. Here, we assume that we have crossing $\left(s_{r}\right)_{i} \times$ $\left(s_{g}\right)_{q}$ that corresponds to a crossing in the front of the respective pseudolines and $\left(s_{r}\right)_{i^{\prime}} \times\left(s_{g}\right)_{q^{\prime}}$ which also produces a crossing in the front of the respective pseudolines. Note that by equation (3), we must have $i-q \equiv w_{g}-w_{r} \bmod 2$ and $i^{\prime}-q^{\prime} \equiv$ $w_{g}-w_{r} \bmod 2$.

By applying equation (2) twice with the assumption that the green pseudoline $j$ is the same for both crossings (so that $\left(P_{g}\right)^{j}$ has two intersections with $\left(P_{r}\right)^{0}$ ), we see that

$$
\begin{aligned}
& 2 j=(i-q)+\left(w_{g}-w_{r}\right) \quad \text { and } \\
& 2 j=\left(i^{\prime}-q^{\prime}\right)+\left(w_{g}-w_{r}\right),
\end{aligned}
$$

SO

$$
i-q=i^{\prime}-q^{\prime}, \text { or } i^{\prime}-i=q^{\prime}-q
$$

That is, if $i-q \equiv i^{\prime}-q^{\prime} \equiv w_{g}-w_{r} \bmod 2$, so that $i^{\prime}=i+t$ and $q^{\prime}=q+t$, then the red and green pseudolines will intersect inadmissibly. In other words, if the difference between the indices of the two crossings is equal then the two crossing pairs are inadmissible.

Most of the rest of the cases follow similarly; the results are listed below, except for the case of front/back and back/front, which requires more careful analysis. 
Case II: front/front and back/back. Here, we need $i-q \equiv w_{g}-w_{r}$ mod 2 and $i^{\prime}-q^{\prime} \equiv$ $w_{g}-w_{r} \bmod 2$; applying equations (2) and (4), inadmissible beams occur if

$$
i^{\prime}+i=q^{\prime}+q .
$$

That is, $i^{\prime}-q^{\prime}=q-i$. If $q-i=t=i^{\prime}-q^{\prime}$, then $q=i+t$ and $q^{\prime}=i^{\prime}-t$.

Case III: front/front and front/back. Here, we need $i-q \equiv w_{g}-w_{r} \bmod 2$ and $i^{\prime}-q^{\prime} \not \equiv w_{g}-w_{r} \bmod 2$; applying equations (2) and (5), inadmissible beams occur if

$$
i^{\prime}-i=q^{\prime}+q-2 b-1 .
$$

Case IV: front/front and back/front. Here, we need $i-q \equiv w_{g}-w_{r} \bmod 2$ and $i^{\prime}-q^{\prime} \not \equiv w_{g}-w_{r}$ mod 2; applying equations (2) and (6), inadmissible beams occur if

$$
i^{\prime}+i-2 b-1=q^{\prime}-q .
$$

Case V: front/back and front/back. Here, we need $i-q \not \equiv w_{g}-w_{r}$ mod 2 and $i^{\prime}-q^{\prime} \not \equiv$ $w_{g}-w_{r} \bmod 2$; applying equation (5) twice, inadmissible beams again occur if

$$
i^{\prime}-i=q^{\prime}-q .
$$

Case VI: front/back and back/front. Here, we need $i-q \not \equiv w_{g}-w_{r} \bmod 2$ and $i^{\prime}-q^{\prime} \not \equiv w_{g}-w_{r} \bmod 2$. Applying equations (5) and (6), it follows that

$$
\begin{aligned}
& 2 j=(i+q)+\left(w_{g}-w_{r}\right)-2 b-1 \quad \text { and } \\
& 2 j=-\left(i^{\prime}+q^{\prime}\right)+\left(w_{g}-w_{r}\right)+2 b+1,
\end{aligned}
$$

so

$$
i+i^{\prime}+q+q^{\prime}=4 b+2 .
$$

However, by construction, $1 \leq i, i^{\prime}, q, q^{\prime} \leq b$ because $i, i^{\prime}, q, q^{\prime}$ index the beam segments, so

$$
i+i^{\prime}+q+q^{\prime} \leq 4 b .
$$

Therefore this case is impossible.

There is one special case that deserves mentioning: Same Index Cross (SIC). Suppose that $i=q$ and $w_{r}=w_{g}$, so that $i-q \equiv 0 \bmod 2$. Then the results of Case 1 (front/front) and Case 2 (back/back) are both satisfied, and moreover, the same value of $j=0$ is produced in both cases. Therefore, a single same-index crossing produces two intersections between $\left(P_{r}\right)^{0}$ and $\left(P_{g}\right)^{0}$, and thus is forbidden when trying to construct pseudoline arrangements. 


\subsection{Two-beam simplicial arrangements}

In order to have a simplicial arrangement of pseudolines formed by the collection of the mirrors and the pseudolines generated by beams, the regions formed between the beams and the bounding mirrors must all be triangular. In the case of only two beams, this, combined with the above constraints, means that there are only a small number of possibilities for beam interaction.

In particular, given a length $b$ red beam, we can work from the initial green ray to the center, determining at each step what the possibilities are for adding the next green beam segment. The red beam intersects the two bounding mirrors alternately; these intersection points form the vertices of the red beam. Assuming that the red beam is fixed, we will determine what is allowable for the green beam.

\subsubsection{Classification of two-beam simplicial arrangements where $w_{g} \neq w_{r}$}

To begin with, we need to determine allowable starting sequences. It suffices to assume that $w_{r}=1$ and $w_{g}=0$, so the initial rays $\left(s_{r}\right)_{1}$ and $\left(s_{g}\right)_{1}$ are determined. The only possibility for the second green segment $\left(s_{g}\right)_{2}$ is that its endpoint intersects the first red vertex (see Figure 5(a),(b)), since any other placement generates a non-triangular region (Figure 5(c) - (f)). (The case where $k$ is odd and $\left(s_{g}\right)_{2} \times\left(s_{r}\right)_{1}$ in its interior is prohibited either because a quadrilateral region is created, if $k=3$, or because there is no way to place the third segment without creating a non-triangular region, sketched in Figure 5(f).) Note that if $k$ is even, the outermost regions (that intersect the line at infinity) are bounded by two pencils of parallel lines which will both intersect at infinity; thus, for arrangements with even $k$, if $w_{g} \neq w_{r}$, then the line at infinity must be included in the arrangement for the arrangement to be simplicial. For odd $k$, the line at infinity may be included, but it does not have to be.

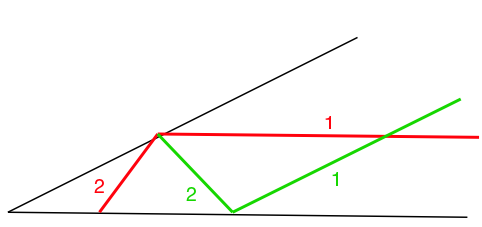

(a)

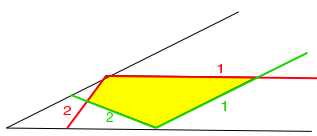

(c)

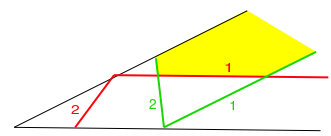

(d)

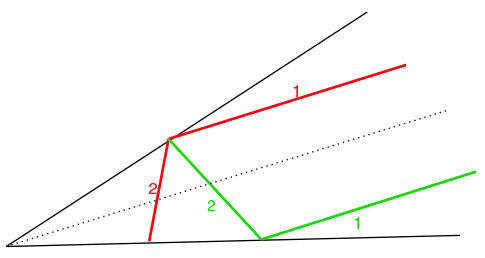

(b)

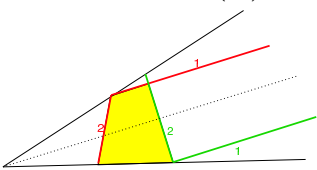

(e)

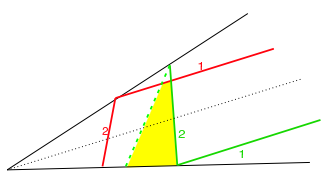

(f)

Figure 5: Placement of the second green segment, when $w_{r} \neq w_{g}$. (a) allowable placement when $k$ is even; (b) allowable placement when $k$ is odd; (c) - (f) inadmissible placement (shaded areas are not triangular) 
Now we need to determine where segment $\left(s_{g}\right)_{3}$ may be placed if it emanates from a red vertex. There are several possibilities, shown in Figure 6.

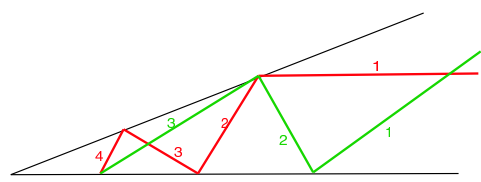

(a)

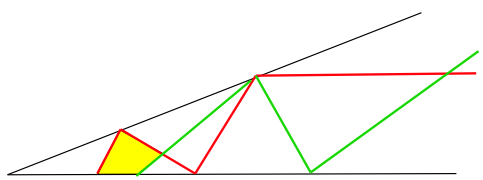

(b)

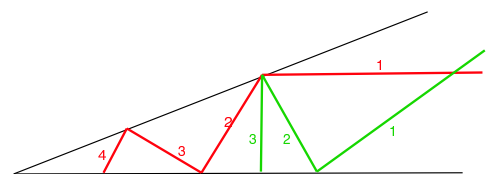

(c)

Figure 6: Placing the third segment. (a) and (b) inadmissible; (c) allowable.

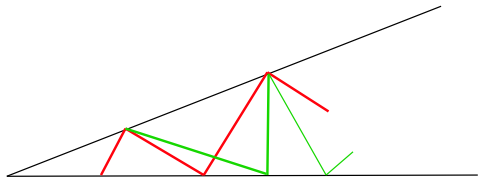

(a)

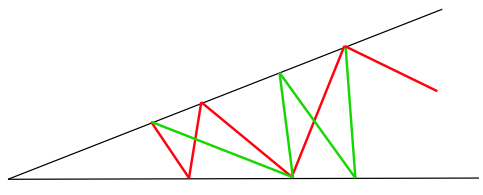

(b)

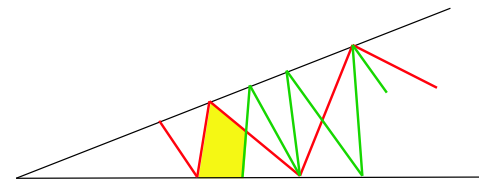

(c)

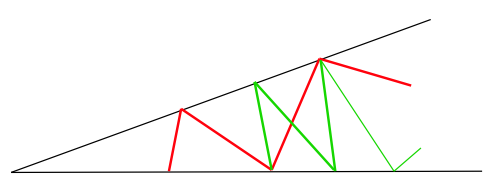

(d)

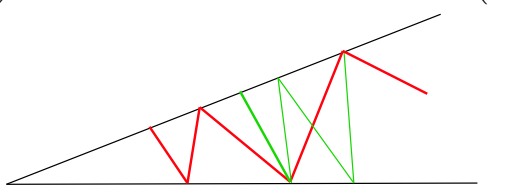

(e)

Figure 7: Placing more segments. (a) - (c) inadmissible placements; (d), (e) allowable placements

Segment $\left(s_{g}\right)_{3}$ cannot connect to the third red vertex (Figure 6(a)) because it is forbidden by Case II, since we have $\left(s_{r}\right)_{1} \times\left(s_{g}\right)_{2}$ and $\left(s_{r}\right)_{4} \times\left(s_{g}\right)_{3}$, and $1+4=2+3$. If $\left(s_{g}\right)_{3}$ crosses $\left(s_{r}\right)_{3}$ in its interior, a non-triangular region will be created (Figure 6 $6(\mathrm{~b})$ ) which cannot be eliminated without creating the inadmissible pattern shown in Figure 7(a). Therefore, the only choice for $\left(s_{g}\right)_{3}$ is that it bounces to ray $r_{0}$ (see Figure 6(c)). (Note that although the figures are shown when $k$ is even, if $k$ is odd, the only change to the figures is in the angle of the initial red and green rays).

When placing the fourth and fifth segments, the only allowable pattern is shown in Figure [7(d) (assuming the thick segments are $\left.\left(s_{g}\right)_{3},\left(s_{g}\right)_{4},\left(s_{g}\right)_{5}\right)$; that is, $\left(s_{g}\right)_{4}$ crosses $\left(s_{r}\right)_{4}$ and $\left(s_{g}\right)_{5}$ bounces to the opposite ray. To see this, note segment $\left(s_{g}\right)_{4}$ cannot connect to the third red vertex (Figure [(a)) because Case I/V forbids it (with $t=2$ ), so it must cross $\left(s_{r}\right)_{4}$ in its interior and then intersect $r_{1}$. But if segment $\left(s_{g}\right)_{4}$ intersects $r_{1}$, then to place $\left(s_{g}\right)_{5}$, we are in the same situation as placing segment $\left(s_{g}\right)_{2}$; any other placement than to the opposite red vertex forms non-triangular regions.

What about placing $\left(s_{g}\right)_{6}$ ? Again, we are placing a segment emanating from a vertex. We need to show that the pattern in Figure $7(\mathrm{~b})$ is inadmissible, but note that Case I/V forbids it with $t=4$. So the only possible placement for $\left(s_{g}\right)_{6}$ is shown in Figure $7(\mathrm{e})$. 
In fact, we can say something stronger, at this point, by using the same analysis used to place segments $\left(s_{g}\right)_{4},\left(s_{g}\right)_{5}$, and $\left(s_{g}\right)_{6}$ repeatedly: the only allowable sequence of segments is formed by requiring the following pattern:

1. if $i \equiv 1 \bmod 3,\left(s_{g}\right)_{i}$ crosses a red segment in its interior and terminates on the opposite bounding mirror (where if $k$ is odd, $\left(s_{g}\right)_{1}$ is taken to be crossing $\left(s_{r}\right)_{1}$, like they do if $k$ is even, although they really are both parallel to the angle bisector of the bounding mirrors);

2. if $i \equiv 2 \bmod 3,\left(s_{g}\right)_{i}$ bounces from the bounding mirror to the opposite red vertex (without intersecting any red segment);

3. if $i \equiv 0 \bmod 3,\left(s_{g}\right)_{1}$ emanates from the red vertex and bounces to the opposite bounding mirror (without intersecting any red ray).

Moreover, this says that this sort of simplicial pseudoline arrangement exists when $b \equiv 0$ or $2 \bmod 3$, since ending the sequence on a segment $\left(s_{g}\right)_{i}$ where $i \equiv 1 \bmod 3$ will yield a non-triangular region (see Figure [7(c)). Examples of pseudoline arrangements when $w_{r} \neq w_{g}$ are shown in Figure 8, In addition, the arrangement shown in Figure 3.15 of [9] (which is also Figure 5.4.2 of [6]) is a two-beam simplicial arrangement with $k=10$ and $w_{r} \neq w_{g}$, and Figure 3.17 of [9] has $w_{r} \neq w_{g}$ and $k=11$; isomorphic versions of these are also shown in Figure 8 .

\subsubsection{Two-beam simplicial arrangements where $w_{g}=w_{r}$}

We again need to begin by determining allowable starting sequences. Without loss of generality, it suffices to assume that $w_{r}=1$ and $w_{g}=1$, so the initial rays $\left(s_{r}\right)_{1}$ and $\left(s_{g}\right)_{1}$ are determined; moreover, we will assume that $\left(s_{g}\right)_{1}$ intersects ray $r_{1}$ farther away from the origin than $\left(s_{r}\right)_{1}$ does. Note that in this case, we have the possibility of Same-Index Crossing (SIC), which is forbidden.

In this case, the placement of $\left(s_{g}\right)_{2}$ and $\left(s_{g}\right)_{3}$ is forced, shown in Figure 9(a); segment $\left(s_{g}\right)_{2}$ cannot be placed intersecting the second red vertex (Figure 9(b)) because SIC forbids it.

If $w_{r}=w_{g}$ and $k$ is odd, then the line at infinity must be excluded from the arrangement; in Figure 9(c), the shaded region is not triangular with the inclusion of the line at infinity. However, if the line at infinity is excluded, the initial placement of beams shown in Figure 9(c) is valid; Figure 10 shows an example of the initial placement of beams with $k=11$ and a sample shaded triangle that is intersected by the (excluded) line at infinity.

Analysis similar to the case when $w_{r} \neq w_{g}$ shows that the only pattern which will generate a simplicial arrangement of pseudolines is the following:

1. $\left(s_{r}\right)_{1}$ and $\left(s_{g}\right)_{1}$ are parallel

2. if $i \equiv 2 \bmod 3,\left(s_{g}\right)_{i}$ crosses a red segment in its interior and terminates on the opposite bounding mirror; 


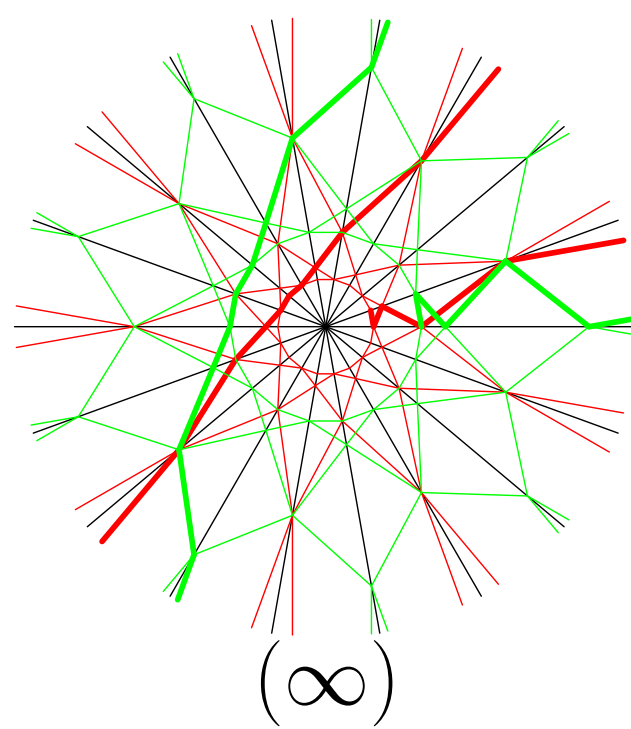

$k=9$

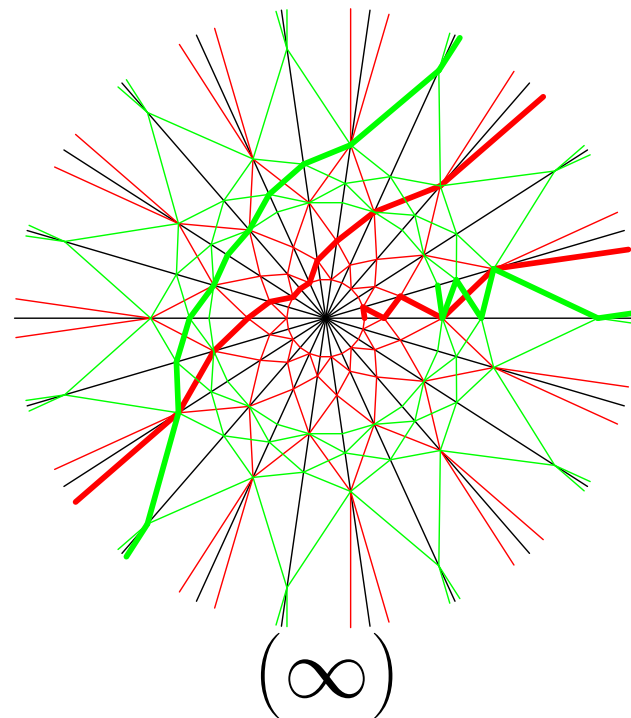

$k=11$

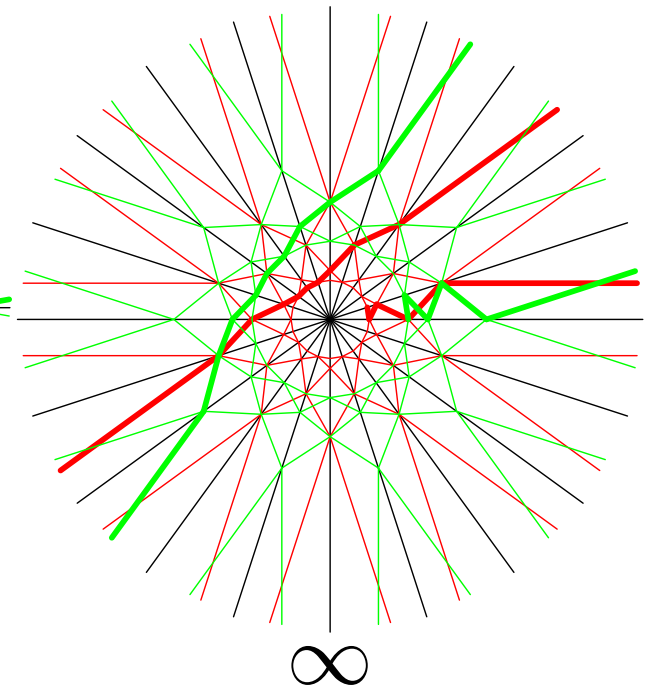

$k=10$

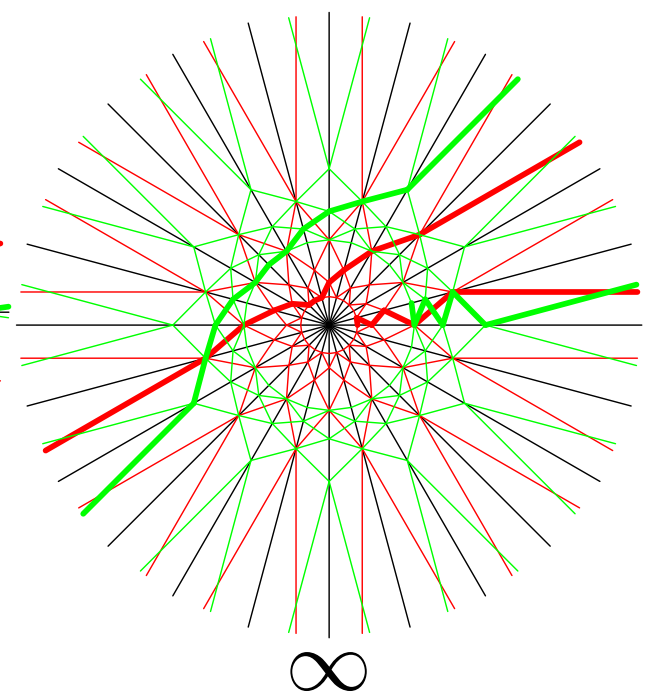

$k=12$

Figure 8: Two-beam simplicial pseudoline arrangement examples, where $w_{r} \neq w_{g}$

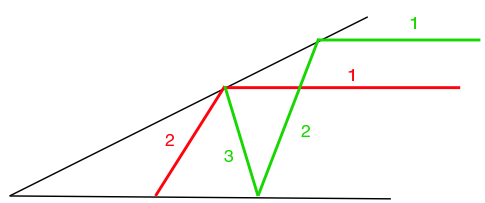

(a)

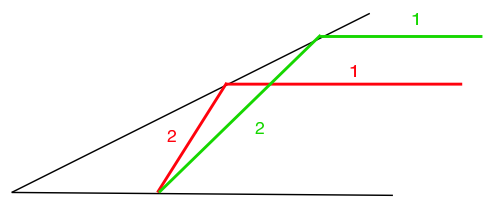

(b)

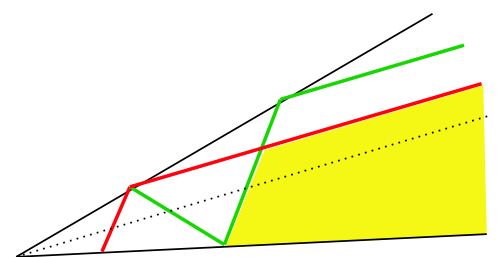

(c)

Figure 9: Potential placement of initial segments when $w_{r}=w_{g}$. 


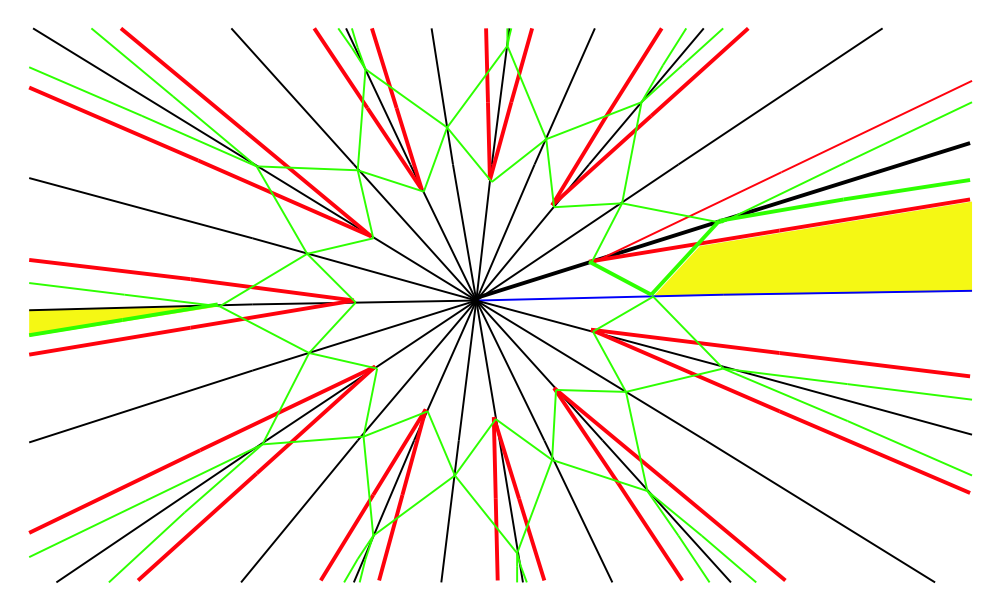

Figure 10: If $w_{r}=w_{g}$ and $k$ is odd, exclusion of the line at infinity allows infinite triangular regions; here $k=11$.

3. if $i \equiv 0 \bmod 3,\left(s_{g}\right)_{i}$ bounces from the bounding mirror to the opposite vertex;

4. if $i \equiv 1 \bmod 3,\left(s_{g}\right)_{1}$ emanates from a vertex and bounces to the opposite bounding mirror.

That is, the pattern of bounces is the same as in the case where $w_{r} \neq w_{g}$, but the index of the corresponding green segment is increased by 1 . In this situation, such arrangements exist if $b \equiv 0$ or $1 \bmod 3$, since $b \equiv 2 \bmod 3$ yields non-triangular regions, as in Figure 7(d). Examples of pseudoline arrangements when $w_{r}=w_{g}$ are shown in Figure [1] addtionally, Figure 3.16 of [9] is a two-beam arrangement with $w_{r}=w_{g}$ and $k=12$.

We collect the previous reasoning into Table 1 and then as a theorem.

Table 1: Valid and invalid two-beam simplicial pseudoline arrangements

\begin{tabular}{|c|c|c|c|c|}
\hline$k \bmod 6$ & $b \bmod 3$ & $w_{r} \neq w_{g}$ OK? & $w_{r}=w_{g}$ OK? & line at $\infty$ allowed? \\
\hline \hline 0 & 0 & yes & yes & yes; required if $w_{r} \neq w_{g}$ \\
\hline 1 & 1 & no & yes & no \\
\hline 2 & 1 & no & yes & yes \\
\hline 3 & 2 & yes & no & yes \\
\hline 4 & 2 & yes & no & required \\
\hline 5 & 0 & yes & yes & only if $w_{r} \neq w_{g}$ \\
\hline
\end{tabular}

Theorem 2. Simplicial arrangements of pseudolines which have the symmetries of a $k$ gon and three symmetry classes of lines generated by two beams of length $\left\lfloor\frac{k+1}{2}\right\rfloor$ and the bounding mirrors exist for all $k$. If $k \equiv 0$ or $5 \bmod 6$, then there are two simplicial 


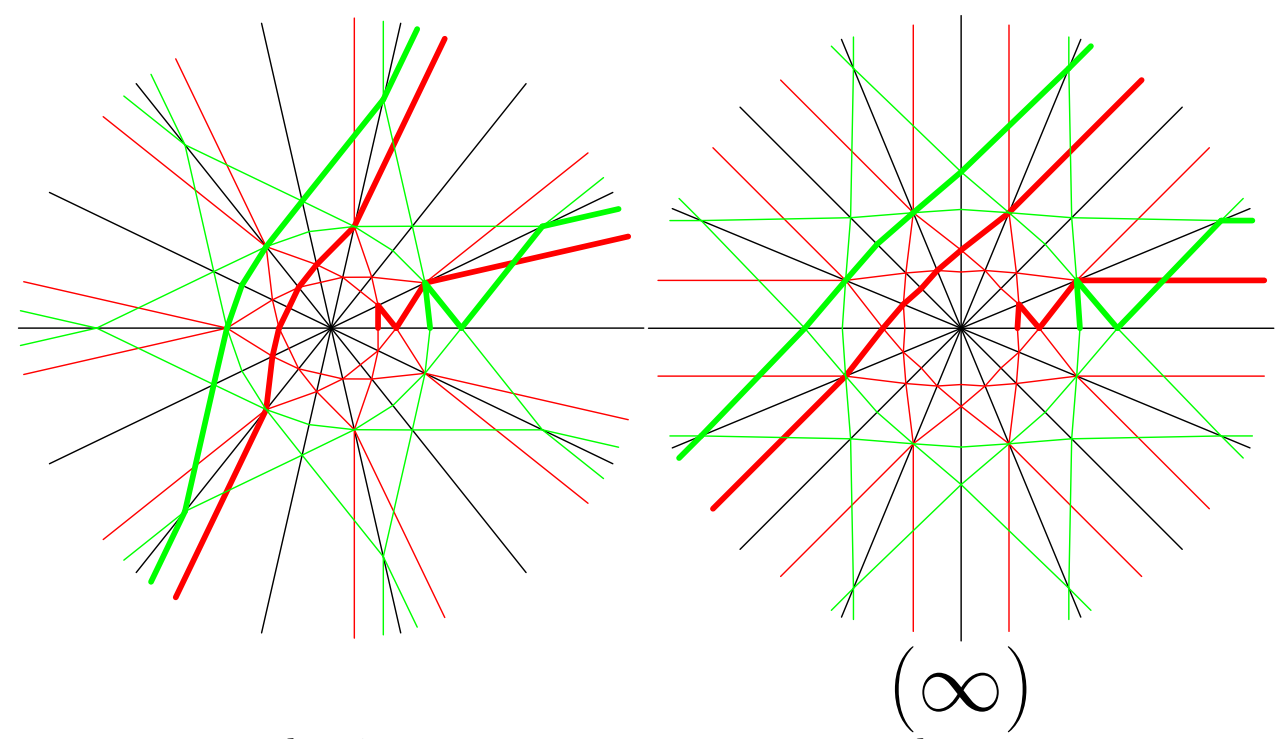

$k=7$

$k=8$
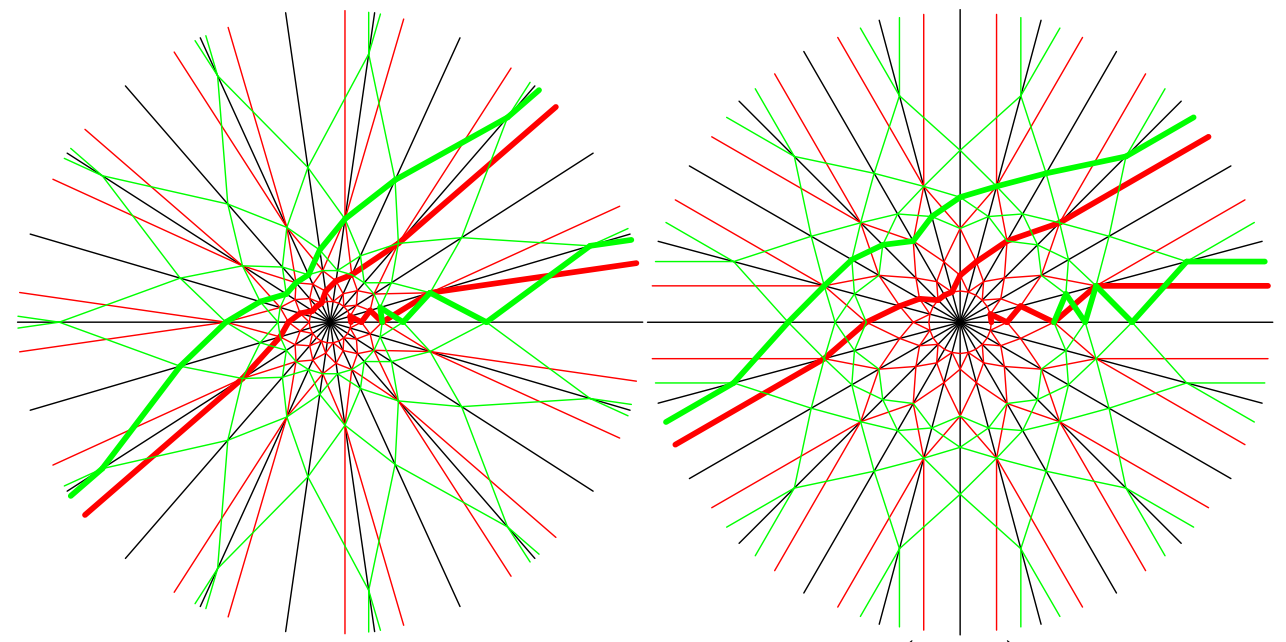

$$
k=11
$$

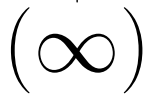

$$
k=12
$$

Figure 11: Two-beam simplicial pseudoline arrangement examples, where $w_{r}=w_{g}$ 
pseudoline arrangments of this sort, one with $w_{r}=w_{g}$ and one with $w_{r} \neq w_{g}$; the second requires the line at infinity to be included in the arrangement, while the first may include the line at infinity if $k$ is even but not if $k$ is odd. If $k \equiv 1$ or $2 \bmod 6$, there exist simplicial arrangements with $w_{r}=w_{g}$; if $k \equiv 1 \bmod 6$ the line at infinity must not be included, but if $k \equiv 2 \bmod 6$ it may or may not be included. If $k \equiv 3,4$, or $5 \bmod 6$ the only two-beam simplicial pseudoline arrangements are with $w_{r} \neq w_{g}$; if $k \equiv 4 \bmod 6$ then the line at infinity must be included in the arrangement, and in the others it may be.

\section{Three-beam simplicial arrangements}

Three-beam simplicial arrangements are considerably more complicated than two-beam simplicial arrangements. This section will present two infinite classes of simplicial threebeam pseudoline arrangements.

\subsection{Interlacing three beams}

One easy class of simplicial three-beam configurations is formed by interlacing two beams (see Figure 121), say red and green, so that all the intersections between red and green segments happen on the interior of the segments (not at a vertex) and then bouncing a third beam in such a way that its initial (pseudo)ray passes through all the interior intersection points of the red and green beams. There are certain adjustments that must be made depending on the parity of $k$. Unlike the two-beam pseudline arrangements, this class of three-beam arrangements requires that the initial ray of the third beam is actually a pseudoray (that is, a curve segment that differs from a ray only in finite part); it will wiggle around connecting the intersection points of the interlaced red and green rays before settling down to be parallel to one of the bounding rays (if $k$ is even) or the angle bisector of the bounding rays (if $k$ is odd).

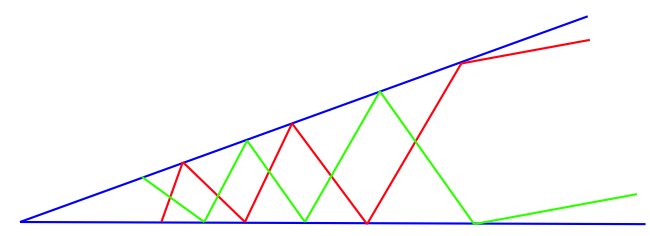

(a)

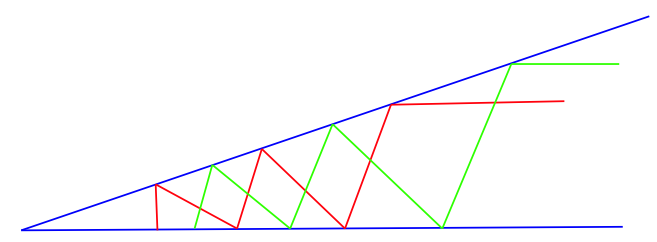

(b)

Figure 12: Interlacing two beams; (a) $k$ is odd; (b) $k$ is even

There are four cases to consider: both $k$ and $b$ are even $(k \equiv 0 \bmod 4) ; k$ is even and $b$ is odd $(k \equiv 2 \bmod 4) ; k$ is odd and $b$ is even $(k \equiv 1 \bmod 4)$; and both $k$ and $b$ are odd $(k \equiv 3 \bmod 4)$.

Each case requires a slight adjustment of the interlacing of the last (closest to the origin) green and red beam segments so that all the regions formed are triangular. In Figure 13 we simply present examples of the beams for the four cases; they generalize in 


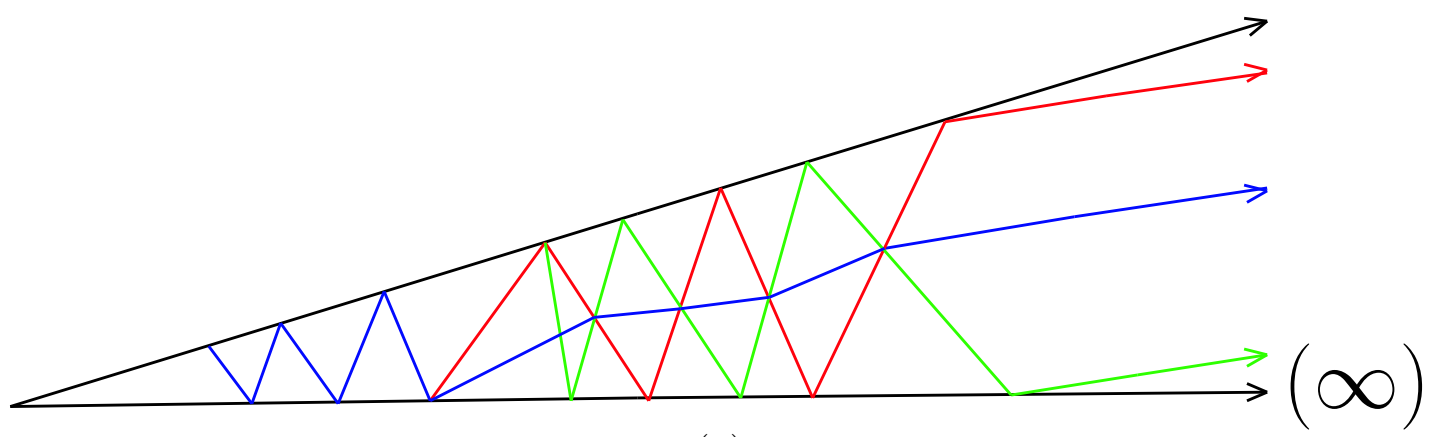

(a)

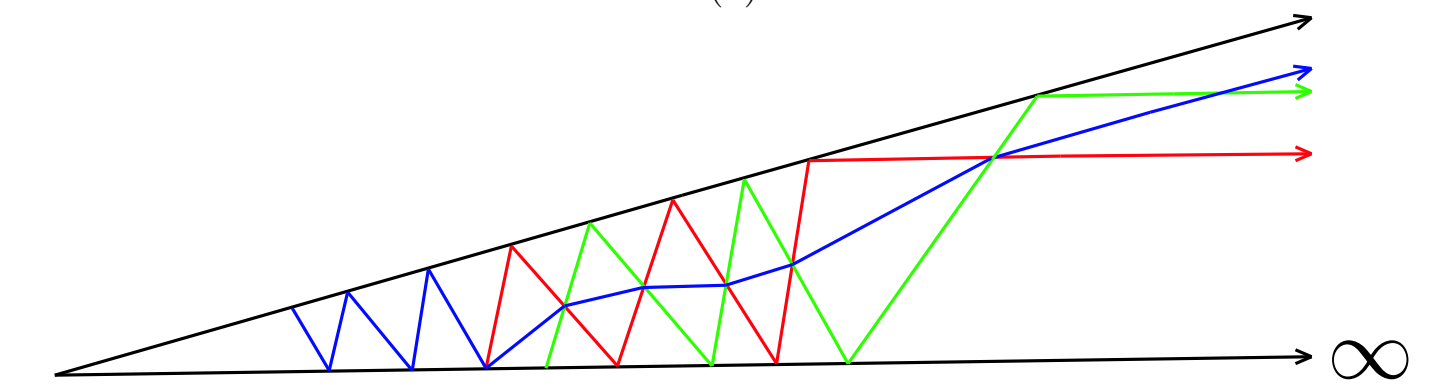

(b)

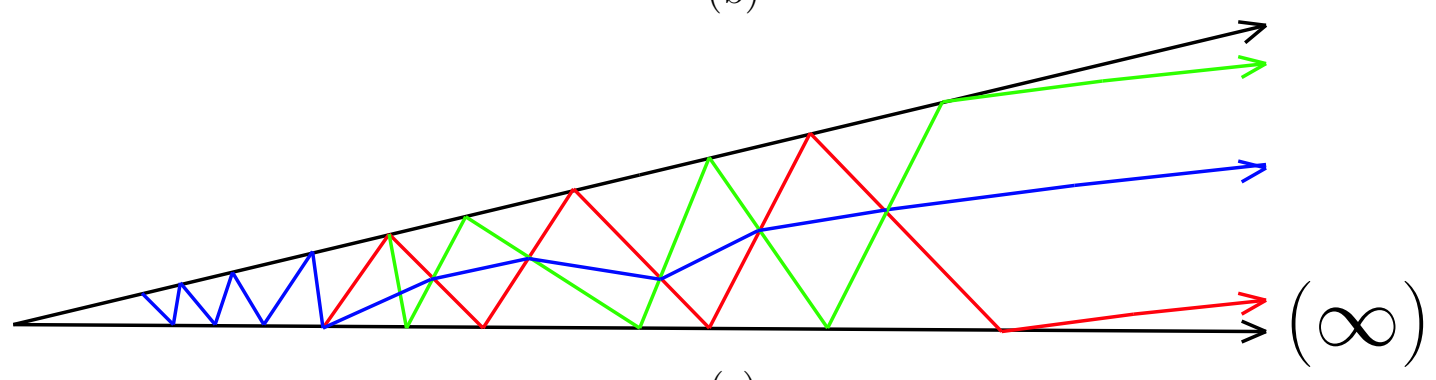

(c)

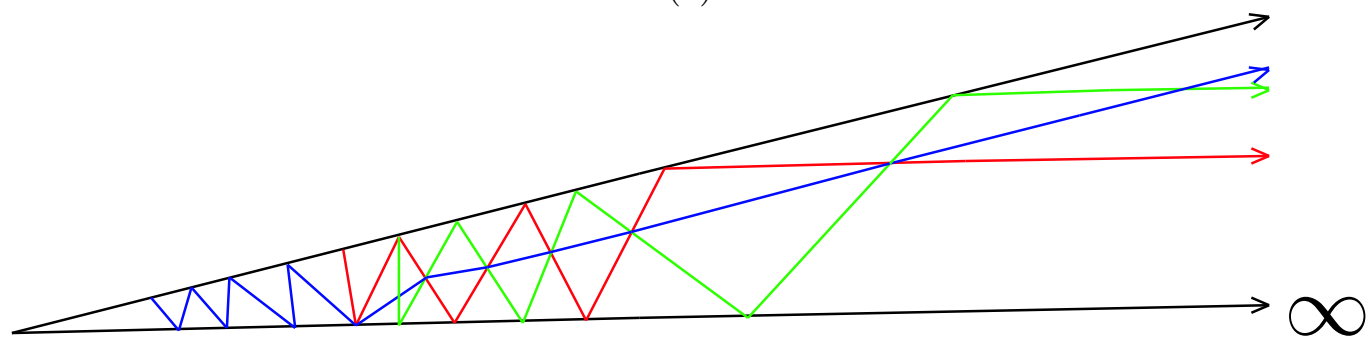

(d)

Figure 13: Examples of the four cases of interlaced three-beam arrangements. (a) $k$ is odd and $b$ is even $(k=11)$ (b) both $k$ and $b$ are even $(k=12)$; (c) both $k$ and $b$ are odd $(k=13) ;(\mathrm{d}) k$ is even and $b$ is odd $(k=14)$. 


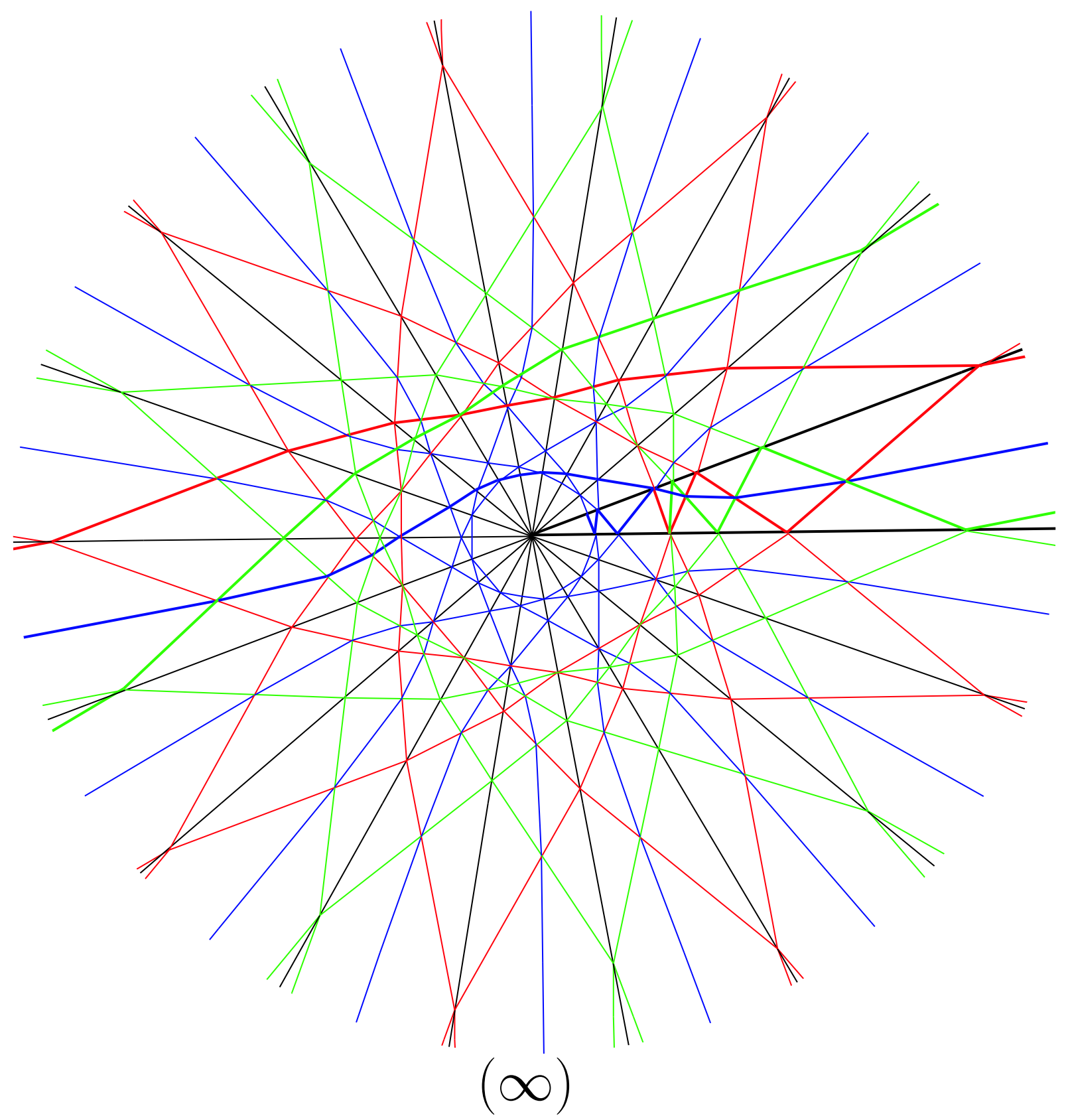

Figure 14: An example of a complete interlaced three-beam arrangement, with $k=9$. 
the obvious way. If $k$ is odd, the initial rays of each of the three beams are parallel to the angle bisector of the bounding rays; if $k$ is even, the interlaced (red and green) beams have initial rays parallel to one of the bounding rays and the third beam has initial ray parallel to the other bounding ray, requiring that the line at infinity is included as a line of the arrangement (since there exist infinite regions that are bounded by two pencils of parallel lines). Figure 14 shows a completed three beam arrangement formed by interlacing, with $k=9$.

\subsection{Three-beam arrangements with diamonds}

While in the interlaced family of three beam arrangements, the third (blue) beam did most of its bouncing near the center of the arrangement, in the second family of threebeam arrangements that will be presented, the blue beam will do most of its bouncing far away from the center. The red and green beams will have a small amount of (modified) interlacing, forming diamonds between the green and red beams, which will be filled in by the bounces of the blue beam, and then will settle down to bouncing in the ways allowed to produce the two-beam arrangements; examples are shown in Figure 15. While the interlaced family of three-beam arrangements also has diamonds formed between the red and green beams, in that family, the blue beam simply crosses the diamonds transversally, while in the diamonds family, there are fewer diamonds and each diamond is 'filled in' by the blue beam bouncing back and forth across it several times. That is, the difference between the two families is in the behavior of the blue beam; the initial behavior of the green and red beams in the two families is quite similar.

Note that in counting diamonds, in the odd case, the outermost region is counted as a diamond, since the initial rays of the outermost region intersect at infinity.

(a)

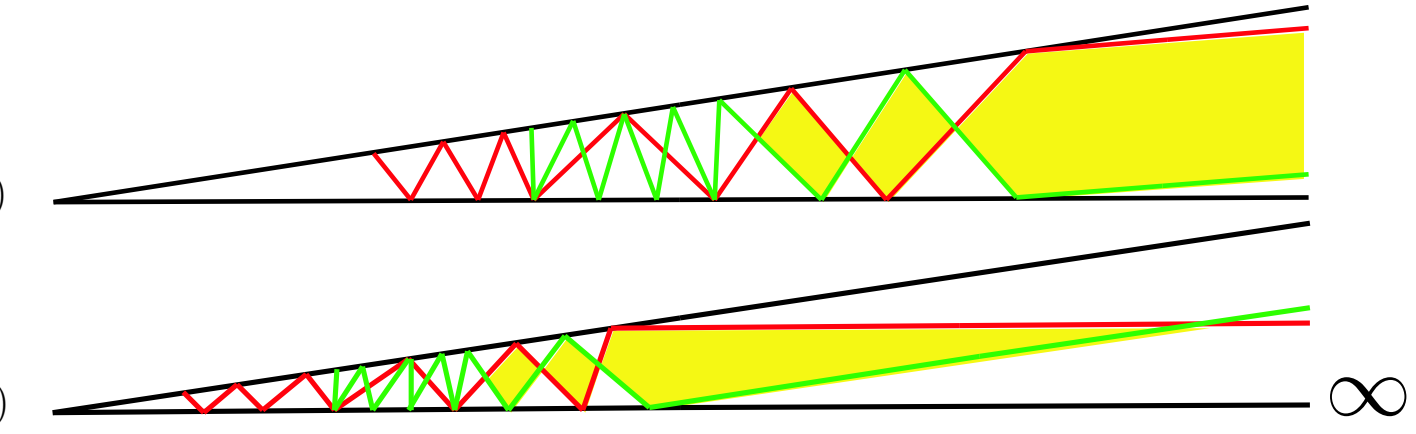

(b)

Figure 15: Interlacing beams to make diamonds (shaded), and then continuing to bounce simplicially. In this figure, $d=3$. (a) $k=21$; (b) $k=22$.

To see how many diamonds should be formed with red and green beams of a given length, we need to analyze how the blue beam can 'fill in' a diamond by connecting its single-color vertices (that is, the vertices that lie on the bounding mirrors). As in the previous families, things work slightly differently when $k$ is odd or $k$ is even, because of the difference in the angle of the initial rays. 


\subsubsection{Diamond analysis: $k$ is odd}

If $k$ is odd, the outermost diamond takes three blue segments to fill in, shown in Figure 16(a). Each diamond takes four blue segments to fill in, from the middle of the previous diamond to the middle of the next diamond (Figure 17(a)). After all the diamonds have been filled in, ending in the middle of the last diamond, either the blue beam can end (Figure 17(b)), or two more segments can be added to end the blue beam at the point of a diamond, shown in Figure 17(c).

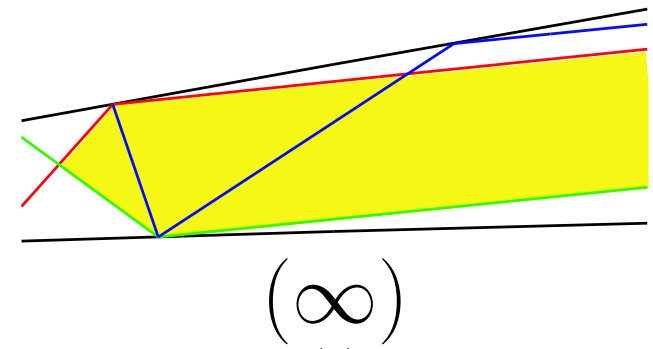

(a)

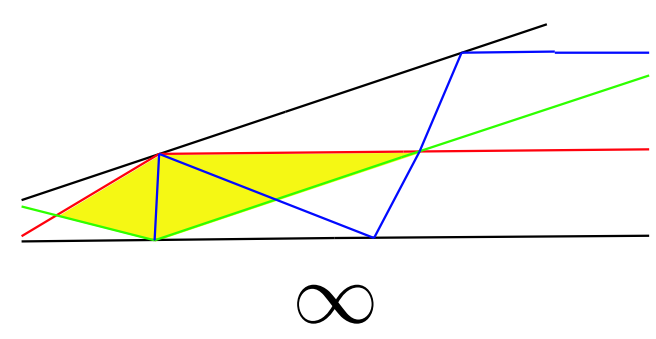

(b)

Figure 16: Filling in the initial diamond. (a) $k$ is odd; (b) $k$ is even.

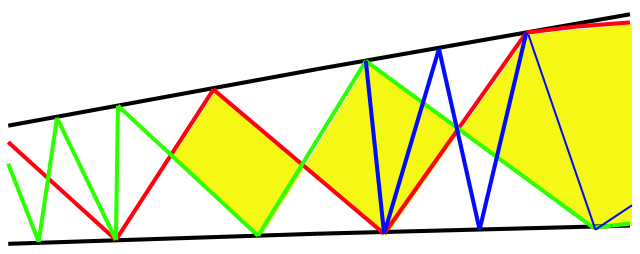

(a)

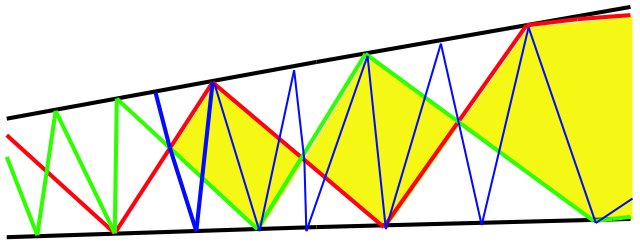

(c)

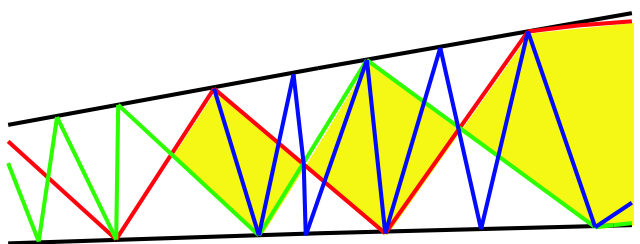

(b)

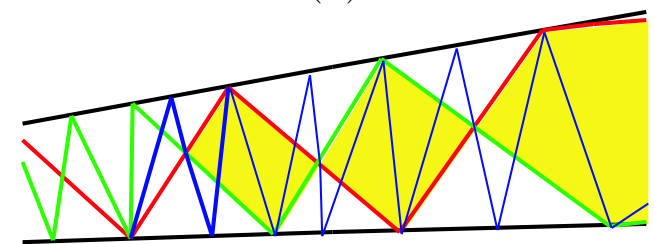

(d)

Figure 17: Filling in diamonds. (a) Filling in an inner diamond takes 4 blue segments (thick); (b) Filling in the last diamond to the middle; (c) Filling in the last diamond to the point takes two extra segments (thick); (d) Filling in the last diamond to the point, plus one extra segment, takes three extra segments (thick).

To determine how many blue segments are needed to fill in $d$ diamonds, if $k$ is odd, it takes 3 blue segments to fill in the initial diamond (to the middle) (Figure 16); in addition, four blue segments are needed to fill in the next $d-1$ diamonds to the middle of the last diamond (Figure 177(a) and (b)), plus, possibly, an additional two segments 
(Figure 17(c)). That is,

$$
\begin{aligned}
& \text { \#blue segments }=3+4(d-1)=4 d-1 \\
& \text { \#blue segments }=3+4(d-1)+2=4 d+1
\end{aligned}
$$

The final observation required is that after the diamonds are constructed and filled in by blue segments, we need the red and green segments to interact as in the two-beam case. In particular, this means that after the diamonds have been constructed, the next green segment must terminate at a red vertex, so that a triangular region is formed. (That is, this segment begins the two-beam simplicial bouncing of the red and green beams.) This transition is shown, for example, in Figure 15. Therefore, it is possible, in the case where two extra blue segments have been added after filling the final diamond, to add a final blue segment, shown in Figure 17(d), so that we also have the following possibility:

$$
\text { \#blue segments }=3+4(d-1)+3=4 d+2
$$

Therefore, given $d$ diamonds, there are three options for the possible number of blue segments needed to fill in those diamonds. To determine which of these options is possible, we need to consider more closely how many red and green segments are needed. At the end of the diamonds, the red and green segments need to interact as discussed in Section 4.2. In particular, the segments need to interact as stated at the end of Section 4.2.1. because by construction the red and green vertices that form the end of the diamonds are on opposite rays. This constrains the number of remaining red and green segments.

The initial diamond takes two green (and red) segments to construct, and each additional diamond takes one more green (and red) segment to construct. Therefore, $2+(d-1)=1+d$ segments have been accounted for. By the discussion in Section 4.2.1. the remaining number $q$ of green (and red) segments must satisfy $q+1 \equiv 0$ or 2 mod 3 (because the $q$ segments we are accounting for do not include the initial ray, but the analysis given in Section 4.2.1 did); that is, $q=3 j+t$ where $t=-1$ or 1 . We can use this count to determine, given $d$, allowable choices for $b$, the total number of blue, red or green segments. There are six cases to check, determined by solving the equation

$$
1+d+3 j+t=4 d+r
$$

where $t=-1,1$ and $r=-1,1,2$. Straightforward algebra and the observation that both $d$ and $j$ must be integers yields the following possibilities:

$$
\begin{array}{rll}
t=1, r=-1 & \text { so } & b=4 d-1 \\
t=1, r=2 & \text { so } & b=4 d+2
\end{array}
$$

Thus, when $k$ is odd, diamond arrangements exist for $b \equiv-1$ or $2 \bmod 4$ and $k=$ $2 b+1$. 


\subsubsection{Diamond analysis: $k$ is even}

If $k$ is even, things proceed almost identically to the case where $k$ is odd, except for the following changes: (i) it takes four blue segments to fill in the first diamond instead of the three in the odd case, shown in Figure 16(b); (ii) the line at infinity must be included as a line of the arrangement, since the interlacing and placement of the initial segments (see Figures 15(b) and 16(b)) forms infinite regions bounded by two pencils of parallel lines. The rest of the arguments for how diamonds are filled in and how segments are counted are still valid. Thus, the possible number of blue segments is

$$
\begin{aligned}
& \text { \#blue segments }=4+4(d-1)=4 d ; \\
& \text { \#blue segments }=4+4(d-1)+2=4 d+2 ; \\
& \text { \#blue segments }=4+4(d-1)+3=4 d+3 ;
\end{aligned}
$$

so equation (8) must be solved where $t=-1,1$ and $r=0,2,3$.

This time, the solutions are

$$
\begin{array}{rll}
t=-1, r=0 & \text { so } & b=4 d \\
t=-1, r=3 & \text { so } & b=4 d+3 \\
t=1, r=2 & \text { so } & b=4 d+2 .
\end{array}
$$

To summarize,

Theorem 3. Diamond arrangements exist for all $b \not \equiv 1 \bmod 4$ and $k=2 b$ and $b \equiv$ -1 or $2 \bmod 4$ and $k=2 b+1$.

Two completed diamond arrangements are shown in Figures 18] and 19, in addition, the arrangement shown in [4] is a diamond arrangement with $k=8$.

\section{Multi-beam arrangements}

Figure 20] shows a (linear) four-beam simplicial arrangement with $k=6$; this is arrangement $\mathcal{A}(30,3)$ in Grünbaum's catalogue [7]. Figure 21 shows a similar four-beam pseudoline arrangement with $k=8$. There are several four-beam (and one five-beam) linear simplicial arrangements, indicated in Table 2, but no general treatment is known. I propose the following:

Conjecture 1. There exist infinite families of p-beam simplicial pseudoline arrangements, for some $p \geq 4$.

It should be noted that there exist sporadic symmetric line and pseudoline arrangements which are not analyzable using the kaleidoskope technique discussed in this paper. Figure 22 shows an arrangement due to Grünbaum that does not obey the beam constraints; however, it may be viewed as a combination of a kaleidoscope arrangement with $k=3$ and a kaleidoscope arrangement with $k=6$. These arrangements and other pseudoline generalizations of known symmetric sporadic linear arrangements will be analyzed in a later study. 


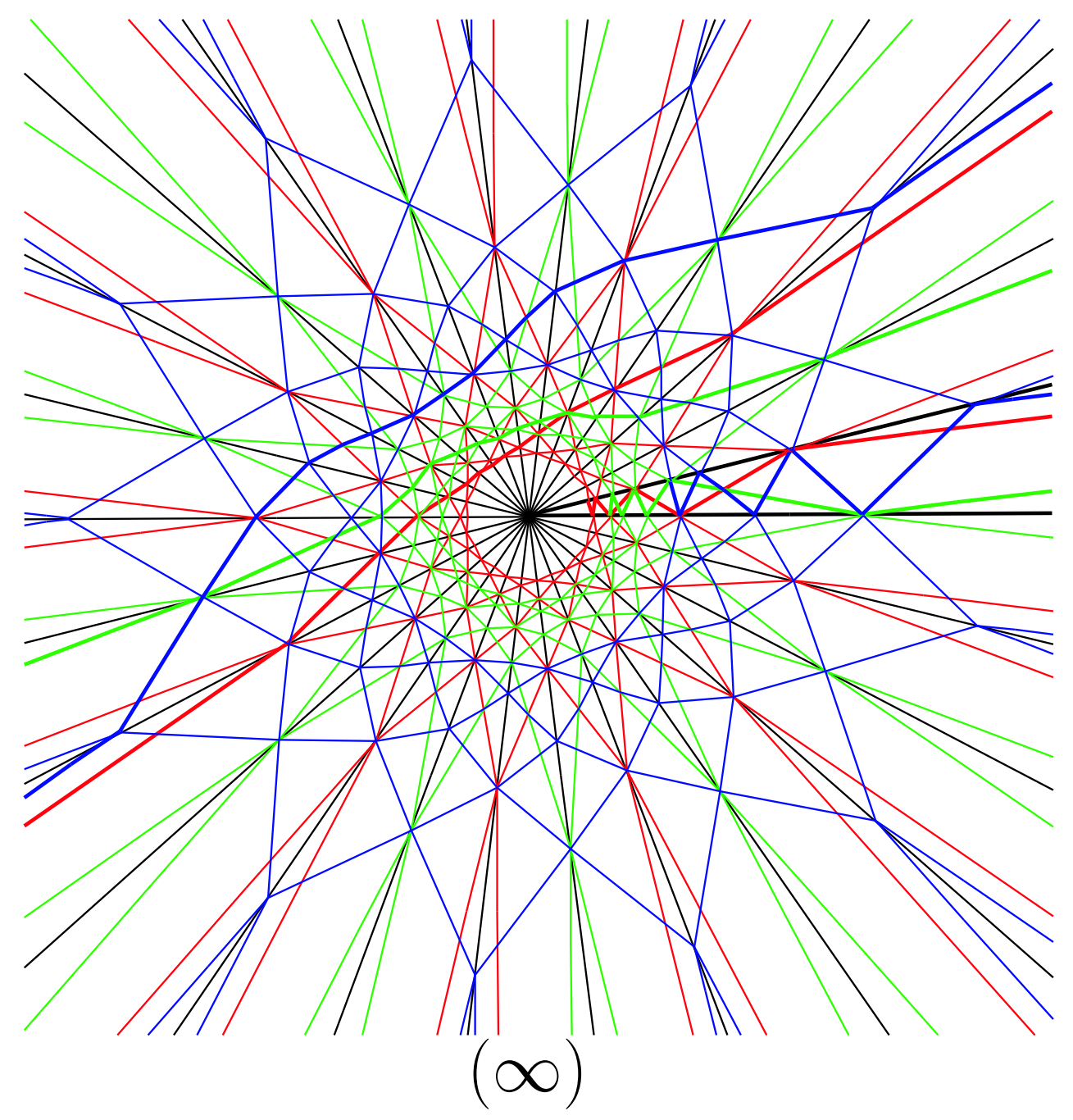

Figure 18: A completed diamond arrangement of type $b=4 d-1$ for $d=2(b=7, k=13)$ 


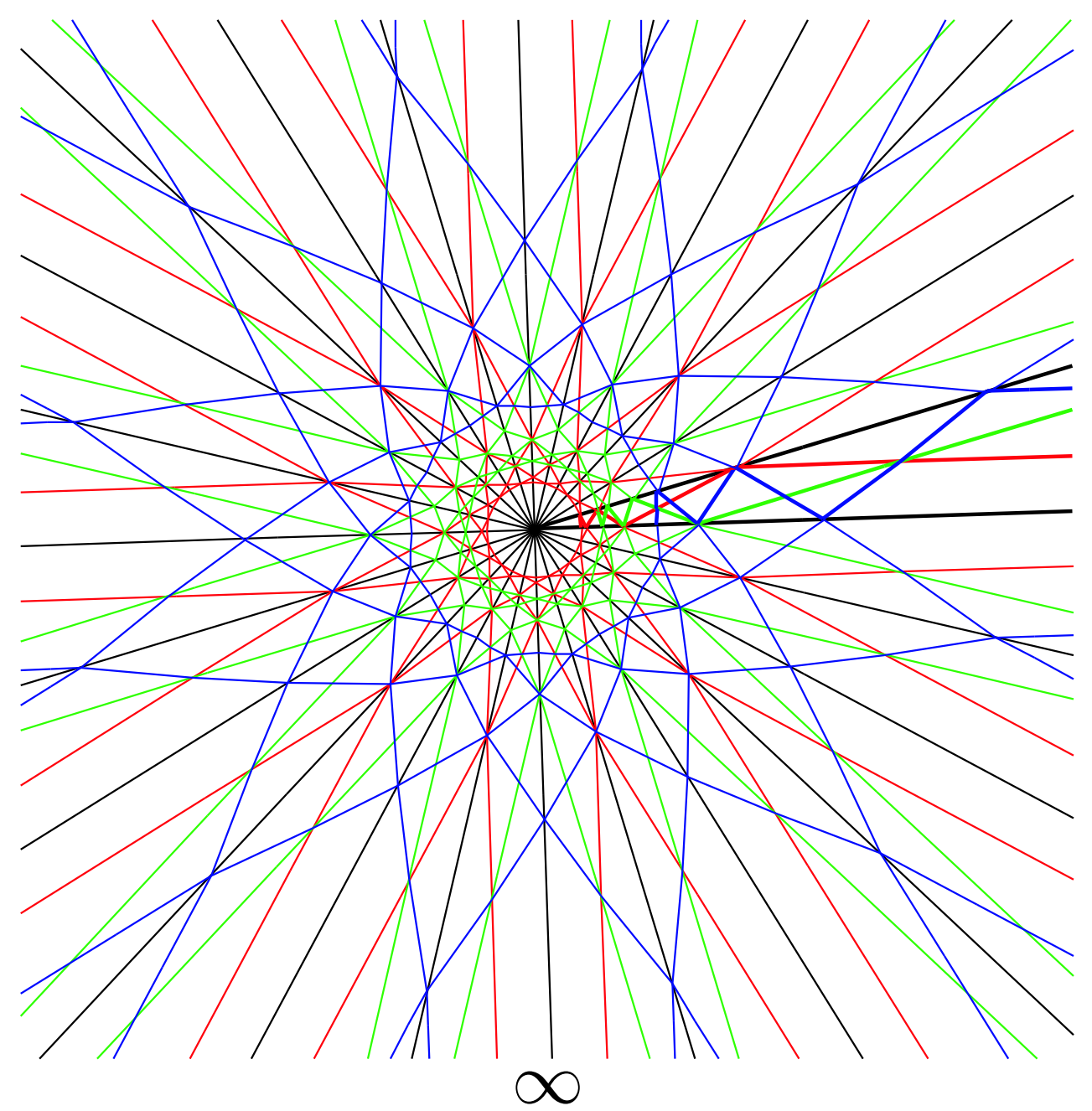

Figure 19: A completed diamond arrangement of type $b=4 d+2$ with $d=1(b=6, k=$ $12)$. 


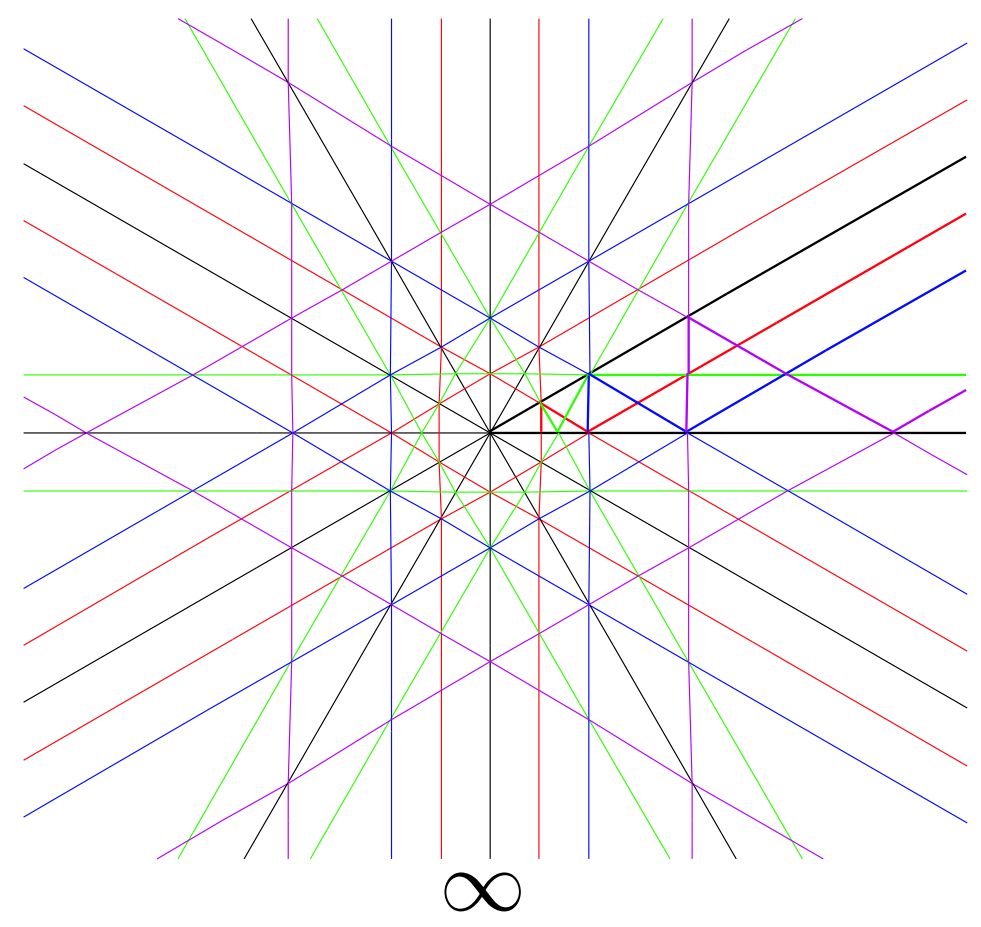

Figure 20: A four-beam simplicial line arrangement, with $k=6$.

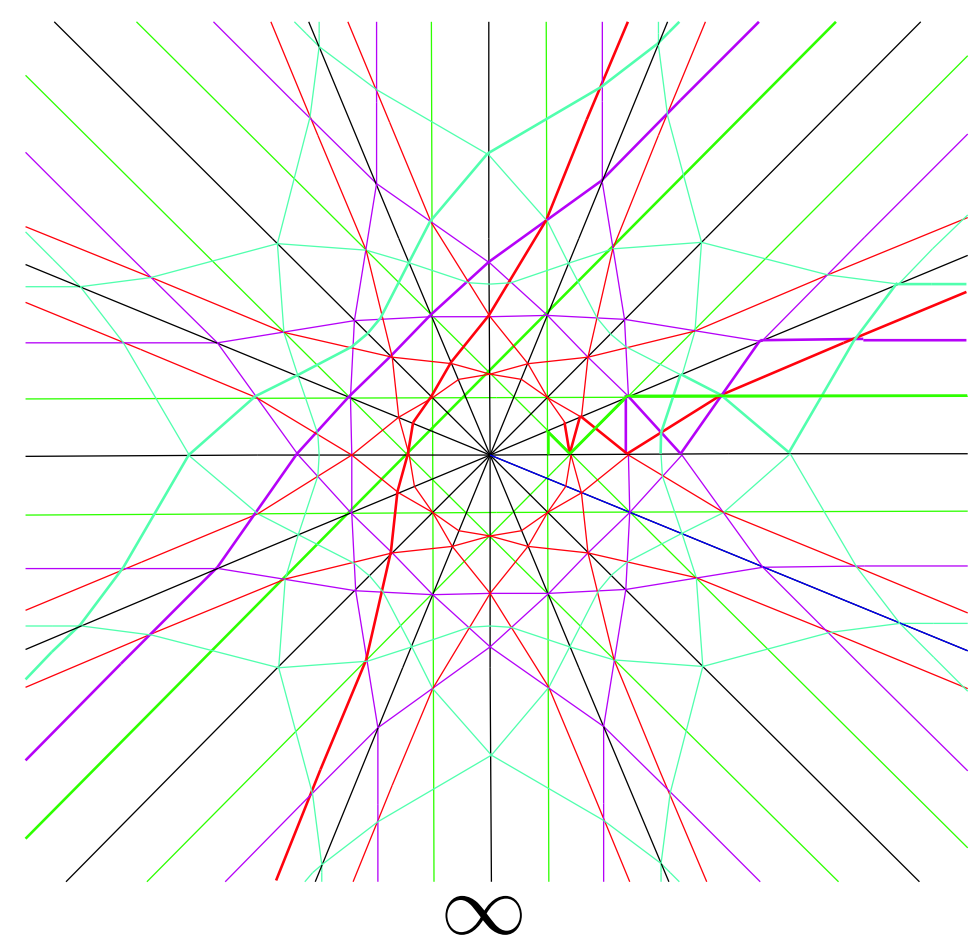

Figure 21: A four-beam simplicial pseudoline arrangement, with $k=8$. 


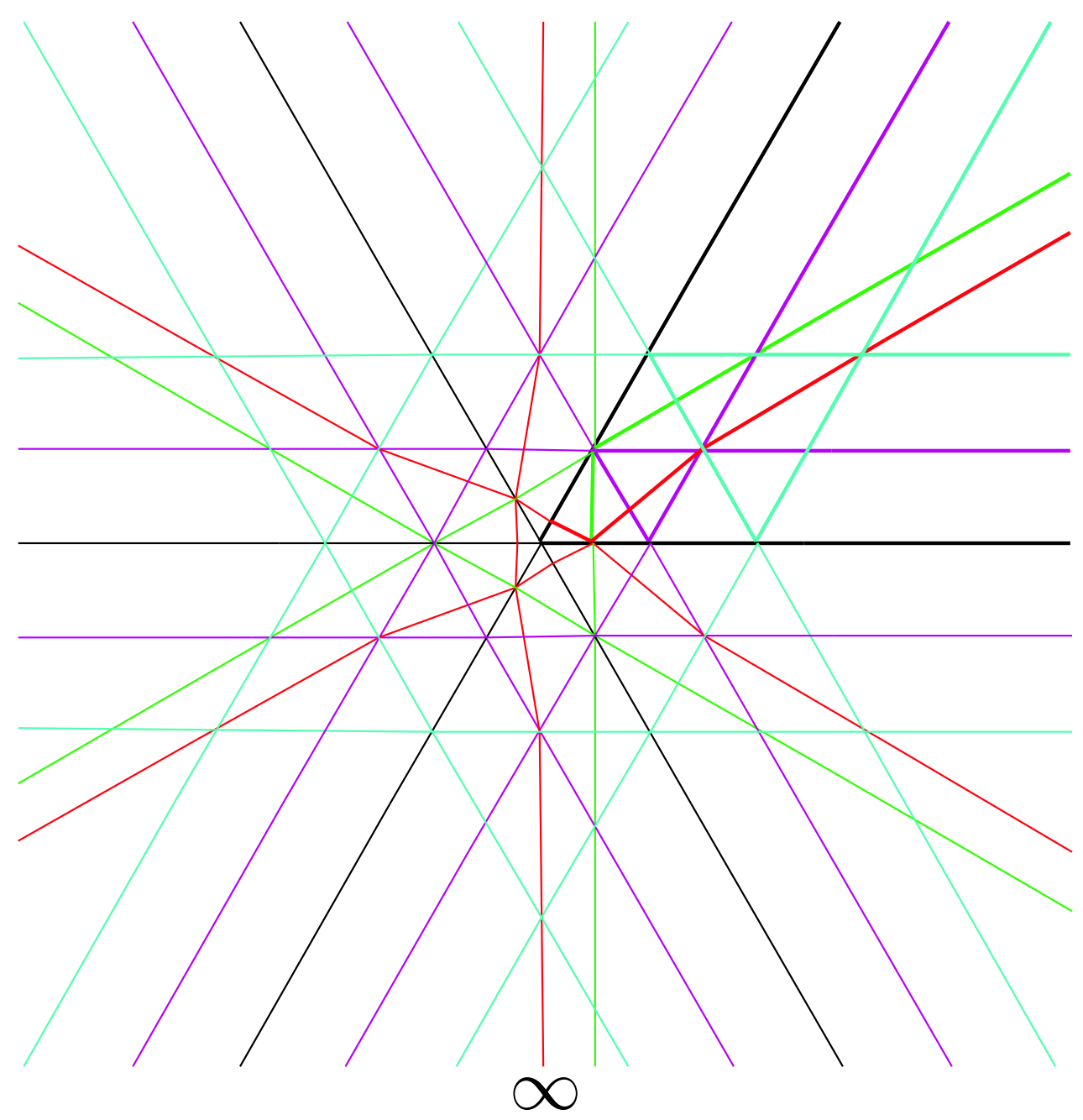

Figure 22: A sporadic symmetric pseudoline arrangement, with $k=3$. 


\section{Linear kaleidoscope arrangements}

Many of the simplicial linear arrangements given in Grünbaum's catalogue of simplicial arrangements [7] may be interpreted as kaleidoscope arrangements. They are listed in Table 2

Table 2: Linear kaleidoscope arrangements, listed by symbol from [7; $k$ is the symmetry of the arrangement and $\infty$ means the line at infinity is included. Where determinable, the type of the arrangement is indicated: $\neq$ indicates a two-beam configuration with $w_{r} \neq w_{g}$; $=$ indicates a two-beam configuration with $w_{r}=w_{g} ; \diamond$ indicates a three-beam diamond arrangement; and $\S$ indicates a three-beam interlaced arrangement.

\begin{tabular}{c|c|c|c|c||c|c|c|c|c} 
arrangement & $k$ & $\#$ beams & $\infty ?$ & type & arrangement & $k$ & $\#$ beams & $\infty$ type \\
\hline $\mathcal{A}(2 k, 1)$ & $k$ & 1 & & & $\mathcal{A}(19,1)$ & 6 & 2 & $\infty$ & $=$ \\
$\mathcal{A}(4 k+1,1)$ & $2 k$ & 1 & $\infty$ & & $\mathcal{A}(19,3)$ & 6 & 2 & $\infty$ & $\neq$ \\
$\mathcal{A}(10,2)$ & 3 & 2 & $\infty$ & $\neq$ & $\mathcal{A}(20,2)$ & 5 & 3 & & $\diamond$ \\
$\mathcal{A}(12,3)$ & 3 & 3 & & & $\mathcal{A}(21,2)$ & 5 & 3 & $\infty$ & $\diamond$ \\
$\mathcal{A}(13,2)^{*}$ & 3 & 3 & $\infty$ & & $\mathcal{A}(24,2)$ & 8 & 2 & & $=$ \\
$\mathcal{A}(13,2)^{*}$ & 4 & 2 & $\infty$ & $\neq$ & $\mathcal{A}(25,3)$ & 5 & 4 & & \\
$\mathcal{A}(15,1)$ & 5 & 2 & & $\neq$ & $\mathcal{A}(25,4)$ & 6 & 3 & $\infty$ & $\S$ \\
$\mathcal{A}(15,3)$ & 3 & 4 & & & $\mathcal{A}(25,5)$ & 8 & 2 & $\infty$ & $=$ \\
$\mathcal{A}(16,3)$ & 3 & 4 & $\infty$ & & $\mathcal{A}(26,2)$ & 5 & 4 & $\infty$ & \\
$\mathcal{A}(16,4)$ & 5 & 2 & $\infty$ & $\neq$ & $\mathcal{A}(30,2)$ & 5 & 5 & & \\
$\mathcal{A}(17,4)$ & 4 & 3 & $\infty$ & & $\mathcal{A}(31,2)$ & 6 & 4 & $\infty$ & \\
$\mathcal{A}(18,2)$ & 6 & 2 & & $=$ & $\mathcal{A}(31,3)$ & 6 & 4 & $\infty$ & \\
& & & & & $\mathcal{A}(37,2)$ & 12 & 2 & $\infty$ & $\neq$
\end{tabular}

* Note that isomorphic representations of $\mathcal{A}(13,2)$ may be analyzed in two ways, as a three-beam arrangement with $k=3$ or as a two-beam arrangement with $k=4$.

\section{Acknowledgements}

The author is extremely grateful to Ursinus College for providing support for this research through its Early Leave Program and to Branko Grünbaum and Gordon Williams for helpful discussions. 


\section{References}

[1] J. C. Arts, B. Grünbaum and J. Llibre. On the number of invariant straight lines for polynomial differential systems. Pacific J. Math. 184(1998), 207-230.

[2] Bokowski, J. and L. Schewe. There are no realizable $15_{4^{-}}$and $16_{4^{-}}$-configurations. Rev. Roumaine Math. Pures Appl. 50 (2005) no. 5-6, 483-493.

[3] Eppstein, David. A kaleidoscope of simplicial arrangements. http://11011110.livejournal.com/18849.html. Retrieved January 22, 2007.

[4] Eppstein, David. Simplicial pseudoline arrangements. http://11011110.livejournal.com/15749.html. Retrieved March 30, 2007.

[5] Eppstein, David. Cubic partial cubes from simplicial arrangements. The Electronic Journal of Combinatorics. 13 (2006) \#R79.

[6] Goodman, Jacob E. Pseudoline arrangements. In Handbook of Discrete and Computational Geometry. CRC Press (1997), 83-109.

[7] Grübaum, Branko. A catalogue of simplicial arrangements in the real projective plane. http://hdl.handle.net/1773/2269, Retrieved on March 30, 2007.

[8] Grünbaum, B. and J. F. Rigby. The real configuration $\left(21_{4}\right)$. J. London Math. Soc (2). 41 (1990), $336-346$.

[9] Grünbaum, B. Arrangements and Spreads. Conference Board of the Mathematical Sciences Regional Conference Series in Mathematics No. 10. American Mathematical Society. (1972)

[10] Grünbaum, Branko. Arrangements of hyperplanes. Proc. Second Louisiana Conf. on Combinatorics, Graph Theory and Computing, R. C. Mullin et al., eds. Louisiana State University, Baton Rouge (1971), pp. 41 - 106. 